\title{
Article \\ Structure, Biosynthesis, and Biological Activity of Succinylated Forms of Bacteriocin BacSp222
}

\author{
Justyna Śmiałek ${ }^{1}$ D , Michał Nowakowski ${ }^{2}$, Monika Bzowska $^{3}$, Oliwia Bocheńska ${ }^{1}$, Agnieszka Wlizło ${ }^{1}$, \\ Andrzej Kozik ${ }^{1}$ (D), Grzegorz Dubin ${ }^{4}$ (D) and Paweł Mak 1,*(D)
}

1 Department of Analytical Biochemistry, Faculty of Biochemistry, Biophysics and Biotechnology, Jagiellonian University, Gronostajowa 7 St., 30-387 Kraków, Poland; justyna.smialek@doctoral.uj.edu.pl (J.Ś.); oliwia.bochenska@gmail.com (O.B.); agnieszka.wlizlo47@gmail.com (A.W.); andrzej.kozik@uj.edu.pl (A.K.)

2 Biological and Chemical Research Centre, Faculty of Chemistry, University of Warsaw, Zwirki i Wigury 101 St., 02-089 Warszawa, Poland; lyam@chem.uw.edu.pl

3 Department of Cell Biochemistry, Faculty of Biochemistry, Biophysics and Biotechnology, Jagiellonian University, Gronostajowa 7 St., 30-387 Kraków, Poland; monika.bzowska@uj.edu.pl

4 Małopolska Centre of Biotechnology, Jagiellonian University, Gronostajowa 7a, 30-387 Kraków, Poland; grzegorz.dubin@uj.edu.pl

* Correspondence: pawel.mak@uj.edu.pl; Tel.: +48-126-646-511

Citation: Śmiałek, J.; Nowakowski, M.; Bzowska, M.; Bocheńska, O.; Wlizło, A.; Kozik, A.; Dubin, G.; Mak, P. Structure, Biosynthesis, and Biological Activity of Succinylated Forms of Bacteriocin BacSp222. Int. J. Mol. Sci. 2021, 22, 6256. https://doi.org/10.3390/ ijms22126256

Academic Editor: Oxana V. Galzitskaya

Received: 20 May 2021

Accepted: 7 June 2021

Published: 10 June 2021

Publisher's Note: MDPI stays neutral with regard to jurisdictional claims in published maps and institutional affiliations.

Copyright: (c) 2021 by the authors. Licensee MDPI, Basel, Switzerland. This article is an open access article distributed under the terms and conditions of the Creative Commons Attribution (CC BY) license (https:/ / creativecommons.org/licenses/by/ $4.0 /)$.

\begin{abstract}
BacSp222 is a multifunctional peptide produced by Staphylococcus pseudintermedius 222 This 50-amino acid long peptide belongs to subclass IId of bacteriocins and forms a four-helix bundle molecule. In addition to bactericidal functions, BacSp222 possesses also features of a virulence factor, manifested in immunomodulatory and cytotoxic activities toward eukaryotic cells. In the present study, we demonstrate that BacSp222 is produced in several post-translationally modified forms, succinylated at the $\varepsilon$-amino group of lysine residues. Such modifications have not been previously described for any bacteriocins. NMR and circular dichroism spectroscopy studies have shown that the modifications do not alter the spatial structure of the peptide. At the same time, succinylation significantly diminishes its bactericidal and cytotoxic potential. We demonstrate that the modification of the bacteriocin is an effect of non-enzymatic reaction with a highly reactive intracellular metabolite, i.e., succinyl-coenzyme A. The production of succinylated forms of the bacteriocin depends on environmental factors and on the access of bacteria to nutrients. Our study indicates that the production of succinylated forms of bacteriocin occurs in response to the changing environment, protects producer cells against the autotoxicity of the excreted peptide, and limits the pathogenicity of the strain.
\end{abstract}

Keywords: bacteriocin; antibacterial; cytotoxic; Staphylococcus pseudintermedius; succinylation; succinylcoenzyme A; nuclear magnetic resonance (NMR); Krebs/tricarboxylic acids cycle

\section{Introduction}

Succinylation is a posttranslational modification based on attachment of the succinic group (3-carboxypropanoyl, - $\mathrm{CO}-\mathrm{CH}_{2}-\mathrm{CH}_{2}-\mathrm{CO}_{2} \mathrm{H}$ ) to the $\varepsilon$-amino group of lysine residues in proteins. The modification was first described as late as in 2011 [1] and due to its widespread distribution in different proteomes, it is attracting increasing attention [2-7]. Succinylation changes the positive charge of the lysine side chain into a negative one. The change radically alters the overall chemical reactivity and introduces a relatively bulky substituent (+100.02 Da mass shift). The side chain of lysine with its primary $\varepsilon$-amino group constitutes reactive centres in a number of enzymes and is involved in many intra- and intermolecular noncovalent interactions. Consequently, succinylation induces significant changes in the function, catalytic properties, structure, and immunogenicity of proteins and influences many different metabolic pathways. Proteomic studies indicate that succinylation is relatively widespread in nature. For example, it involves 738 different 
proteins in human HeLa cells, 844 proteins in human gastric cancer cells, 861 proteins in mouse liver, 184 proteins in mouse heart, or 990 proteins in E. coli $[5,7]$.

Succinylation occurs predominantly via a nonenzymatic mechanism where proteins are modified by succinyl coenzyme A (suc-CoA), a common metabolite in the tricarboxylic acid (TCA) cycle. Suc-CoA is a highly reactive thioester, and its reactivity is a consequence of the ability to form cyclic succinic anhydride intermediate reacting with primary amines in a dose- and $\mathrm{pH}$-dependent manner [8]. Effective succinylation of proteins by suc-CoA is a further consequence of the high level of this metabolite in cells (100-600 nM in eukaryotic mitochondria and ca. $100 \mathrm{nmol} \mathrm{g}^{-1}$ in bacterial cells) [7,9]. The level of succinylated proteins in certain cells results from the equilibrium between the activity of enzymes catalysing suc-CoA formation and enzymes that desuccinylate proteins. The former group of enzymes is represented by a critical constituent of the TCA cycle-an $\alpha$-ketoglutarate dehydrogenase complex (KGDHC) and, to a lesser degree, succinyl-CoA ligase (or succinylCoA synthetase, SCS) as well as carnitine palmitoyltransferase 1A (CPT1A) [7]. All these enzymes regulate the level of succinylated proteins by maintaining the level of cellular suc-CoA. In turn, the most common enzyme that is able to desuccinylate proteins is Sirtuin 5 (SIRT5), which is active both in bacteria and in eukaryotic cells [10]. The enzyme removes the succinyl group in an $\mathrm{NAD}^{+}$-dependent manner, yielding a free lysine side chain, nicotinamide, and succinyl-ADP-ribose [2].

A large number of comprehensive studies address the influence of succinylation on the complex proteomes in different cells, tissues, diverse metabolic pathways, diseases, and microorganisms $[2,3,5-7,11-17]$. At the same time, there are limited numbers of publications that focus on the influence of succinylation on the structure and functions of particular proteins. These studies are primarily focused on evaluation of the effects of in vitro succinylation on such proteins as bovine serum albumin, $\gamma$-globulin, lysozyme, and glutamate dehydrogenase $[8,18,19]$. The influence of physiological succinylation on the structure and activity of a functional protein was most extensively studied in the case of $\alpha \mathrm{B}$-crystallin from the human lens [20]. The present work reports investigation of the structure, biological activity, and mechanism of succinylation of bacteriocin BacSp222. This 50-amino acid long peptide is produced and excreted into the culture medium by a commensal bacterium from dog skin Staphylococcus pseudintermedius strain 222 [21]. The peptide is encoded on a $15 \mathrm{~kb}$ p222 plasmid, does not contain cysteine, is rich in tryptophan, lysine, and arginine residues, and has formylated methionine at the $\mathrm{N}$-terminus (Figure 1). The sequence of BacSp222 does not exhibit significant similarities to other known proteins. However, our earlier nuclear magnetic resonance (NMR) investigation of its structure documented close similarity of BacSp222 to four bacteriocins: aureocin A53, lacticin Q, entrocin $7 \mathrm{~A} / \mathrm{JSA}$, and enterocin 7B/JSB. The similarity and the particular structural features of the BacSp222 molecule allowed us to distinguish a new four-helix bundle type of bacteriocins belonging to subclass IId of bacteriocins from Gram-positive bacteria, comprising linear non-pediocin-like peptides [22].

Bacteriocins are defined as ribosomally synthesized peptides or proteins produced by bacteria and capable of killing phylogenetically related strains at very low concentrations [23]. BacSp222 affects a broad range of staphylococci at micromolar concentrations by forming pores in their membranes, most probably via a barrel-stave mechanism [24]. Most interestingly, the biological role of BacSp222 also involves features typical for virulence factors, i.e., a group of molecules produced by pathogenic microorganisms facilitating the replication and dissemination within the host by subverting or eluding defence mechanisms [25]. In particular, BacSp222 is cytotoxic to many eukaryotic cells, including human skin fibroblasts and keratinocytes as well as murine monocytes and macrophages.

Moreover, at low concentrations (in a nM range), BacSp222 efficiently enhances interferon gamma-induced nitric oxide (NO) release in macrophage-like cell lines. In consequence, immunomodulatory activity of the peptide has been suggested, as NO is a multipotent factor inhibiting leukocyte infiltration and proliferation and regulating cytokine expression and cyclooxygenase activity [21]. Other immunomodulatory activities of 
BacSp222 suggest protein receptor-dependent action, which is presently being investigated in a separate study.

In the present work, we show that BacSp222 is secreted by Staphylococcus pseudintermedius 222 in several forms modified by succinylation. A detailed analysis of the structure, biological activity, and mechanism of post-translational modification of these peptides is reported.

\section{Results and Discussion}

2.1. Staphylococcus pseudintermedius 222 Produces Native BacSp222 and Its Two Posttranslationally Modified Forms: Suc-K20-BacSp222 and suc-K11/K20-BacSp222

The RP-HPLC separation of the $S$. pseudintermedius 222 post-culture medium revealed the main peak of bacteriocin BacSp222 and two additional compounds (No. 2 and 3; Figure 1, the peaks are indicated by red and green arrows). The corresponding compounds were purified to homogeneity using a second RP-HPLC step. The molecular masses were $5921.9,6020.3$, and 6120.2 Da, respectively, for BacSp222 and compounds No. 2 and 3, showing an equal ca. 99-Da mass increase between each form. The mass spectrometry analyses of tryptic peptide fragments of the compounds of interest revealed modifications in one tryptic peptide (residues 14-25, sequence ALYNWAKSHVGK) in compound 2 and in two tryptic peptides (residues 7-13, sequence FLLSKGR and residues 14-25, sequence ALYNWAKSHVGK) in compound 3. The molecular masses of peptides 7-13 and 14-25 were determined as $919.54 \mathrm{Da}$ and $1472.79 \mathrm{Da}$. The comparison with the theoretical mass of the respective peptides, i.e., $819.51 \mathrm{Da}$ and $1372.74 \mathrm{Da}$, indicates a 100-Da increase in each case. The comparison of the MS/MS and MS 3 spectra confirmed the modification at lysine residues, particularly K5 in peptide FLLSKGR and K7 in peptide ALYNWAKSHVGK. The determined mass shift was used to deduce the possible modification. Based on the annotation from Unimod, an online database of protein modifications for mass spectrometry (http:/ / www.unimod.org/, accessed on 20 May 2016), and Zhinong Zhang et al. [1], two most probable modifications were chosen for further evaluation: succinylation or addition of a methylmalonyl group. Since no significant neutral loss of $\mathrm{CO}_{2}(-44 \mathrm{Da})$ for single charged ions nor -22 Da for double-charged ions was observed, as would be expected for methylmalonyllysine, we concluded that succinylation was the modification found in the BacSp222 variants. Overall, the mass spectrometry analyses identified compound 2 as a BacSp222 molecule succinylated at lysine 20 (suc-K20-BacSp222), and compound 3 was identified as a BacSp222 molecule succinylated at lysines 11 and 20 (suc-K11/K20-BacSp222).

These conclusions were independently verified by analysing tryptic peptide fragments of all forms of BacSp222 using automated protein sequencing. The chromatogram corresponding to the sequencing cycle of residue 20 (peptide 14-25 from suc-K20-BacSp222) and from the sequencing cycles corresponding to residues 11 and 20 (peptides 7-13 and 14-25 obtained from suc-K11/K20-BacSp222) revealed an unusual amino acid peak with retention time corresponding to the synthetic $\mathrm{N}-\varepsilon$-succinyl lysine standard (Supplementary Materials Figure S1). This finding supports the conclusion that S. pseudintermedius 222 produces unmodified native BacSp222 bacteriocin and two succinylated forms: suc-K20-BacSp222 and suc-K11/K20-BacSp222. 


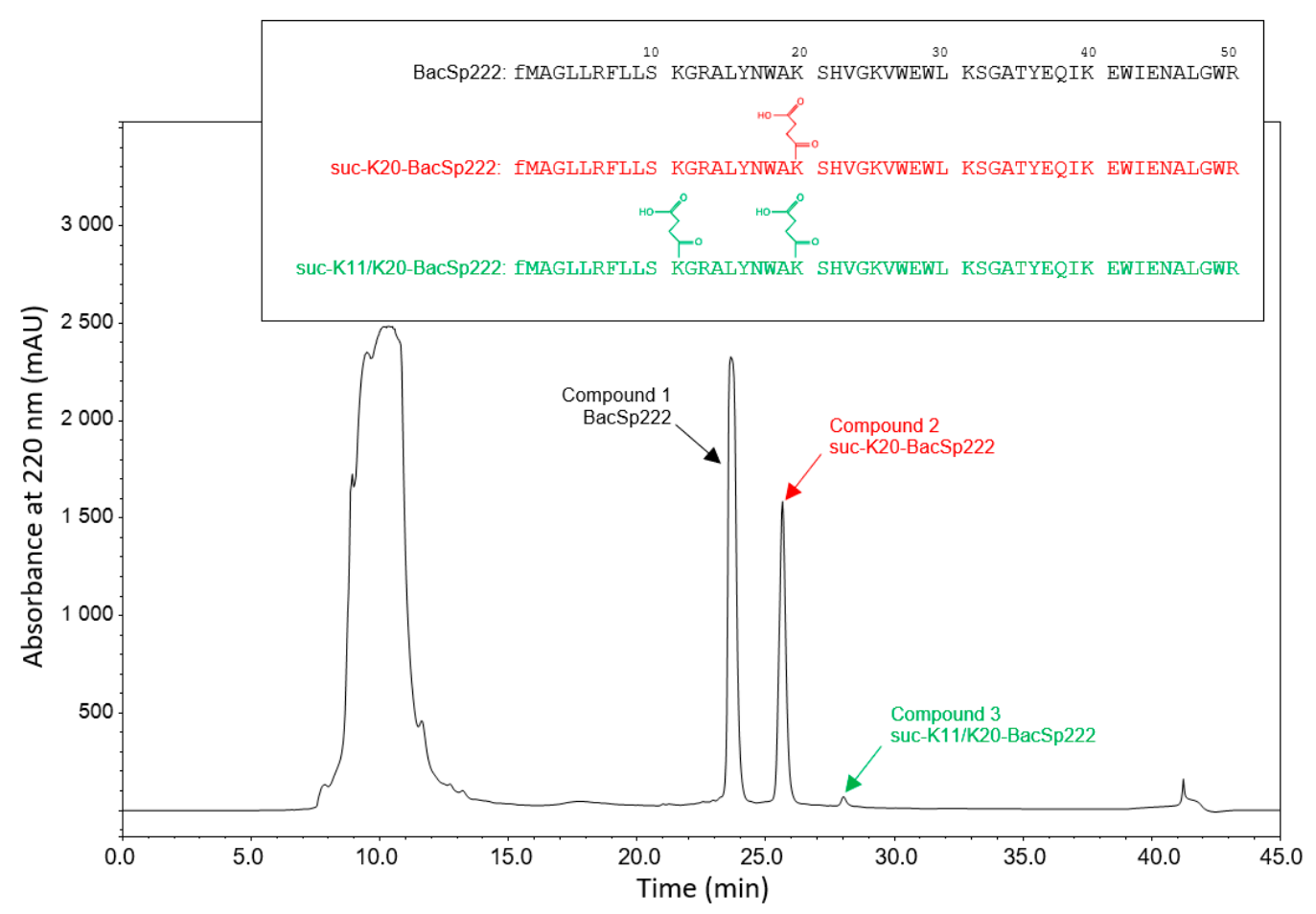

Figure 1. RP-HPLC chromatogram from purification of BacSp222 and its succinylated forms (denoted by black, red and green characters, respectively). The peptide sequence and the succinylation places are presented in the insert. $\mathrm{fM}$ denotes the formylated methionine residue at the $\mathrm{N}$-terminus.

Secretion of factors from cells requires transmembrane transport. Bacteria are equipped with a variety of protein secretion systems. Two types dominate the secretion of bacteriocins by Gram-positive bacteria: the general secretion system (Sec) or the Twin-Arginine Transport (Tat) pathway [26]. Both these systems require a secretion signal in the exported molecule, while BacSp222 is not equipped with such a propeptide, as we have shown earlier [21]. Other authors have suggested the involvement of the ABC-type multidrug resistance transporter protein LmrB in the export of such leaderless bacteriocins [27]. In this study, we tested whether the excretion of BacSp222 and its modified forms was related to production of extracellular vesicles. Post-culture medium deprived of bacterial cells was fractionated using a 100-kDa cut-off membrane, and then the BacSp222 forms were determined both in the presumed vesicle-containing fraction and in the low-molecularweight filtrate. The results clearly show that all BacSp222 forms are contained in the soluble fraction but not in the vesicles. Simultaneously, we could not detect any bacteriocin form in the bacterial cytoplasm, which suggested rapid export of these peptides immediately after synthesis. The mechanisms involved remain to be determined.

Identification of lysines 11 and 20 as sites of succinylation raises a question of why the other lysine residues in the BacSp222 molecule (K25, K31, and K40) do not undergo such modification. Numerous studies mapping thousands of succinylation sites in proteomes of different microorganisms and in animal and human tissues contributed to identification of succinylation patterns and development of machine learning algorithms to predict succinylation sites in particular proteins based on their amino acid sequences, amino acid properties, and theoretical spatial arrangement of amino acid residues [28-34]. We evaluated the following tools: GPSuc [31], iSuc-PseAAC [34], iSuc-PseOpt [30], pSuc-Lys [29], SuccFind [28], and SuccinSite [32]. However, the results poorly match our experimental findings. Only three algorithms (iSuc-PseOpt, pSuc-Lys, and iSuc-PseAAC) indicated K20 as a potential succinylation site. The most frequently indicated site of succinylation was K30 (indicated by four algorithms), while two other algorithms indicated K40. The inconsistency between the prediction and the experimental data and between the different algorithms demonstrates considerable lack of understanding of the determinants 
of succinylation site selection. The algorithms clearly require further improvement to take into account the structure of considered proteins and include more information on species-specific factors.

\subsection{Succinylation Does Not Significantly Influence the Overall Structure of BacSp222}

The influence of succinylation on the bacteriocin structure was investigated using suc-K20-BacSp222 and two complementary techniques-NMR and circular dichroism (CD) spectroscopy. The overlay of the ${ }^{1} \mathrm{H}-{ }^{15} \mathrm{~N}$ HSQC spectra of the native and modified BacSp222 (Figure 2B) demonstrates that succinylation of lysine 20 does not significantly influence the backbone chemical shifts, with the exception of L8 and E37. The significant changes in chemical shifts for those residues may not be explained by the proximity of the modification site. The overlay of the ${ }^{1} \mathrm{H}-{ }^{13} \mathrm{C}$ HSQC spectra tuned to aromatic carbons (Figure 2C) demonstrates that resonances characteristic for residues buried in the hydrophobic core of the peptide remain mostly unaffected. The chemical shift assignment of succinylated BacSp222 was based on chemical shifts obtained previously for an unmodified peptide (BMRB accession number 34044) and carefully verified by analysis of a set of 2D homoand heteronuclear NMR spectra. $80 \%$ of chemical shifts were assigned $(75 \%$ of backbone atoms, $79 \%$ of protons and carbons of aliphatic side chains, and $93 \%$ of protons, nitrogens, and carbons of aromatic side chains).
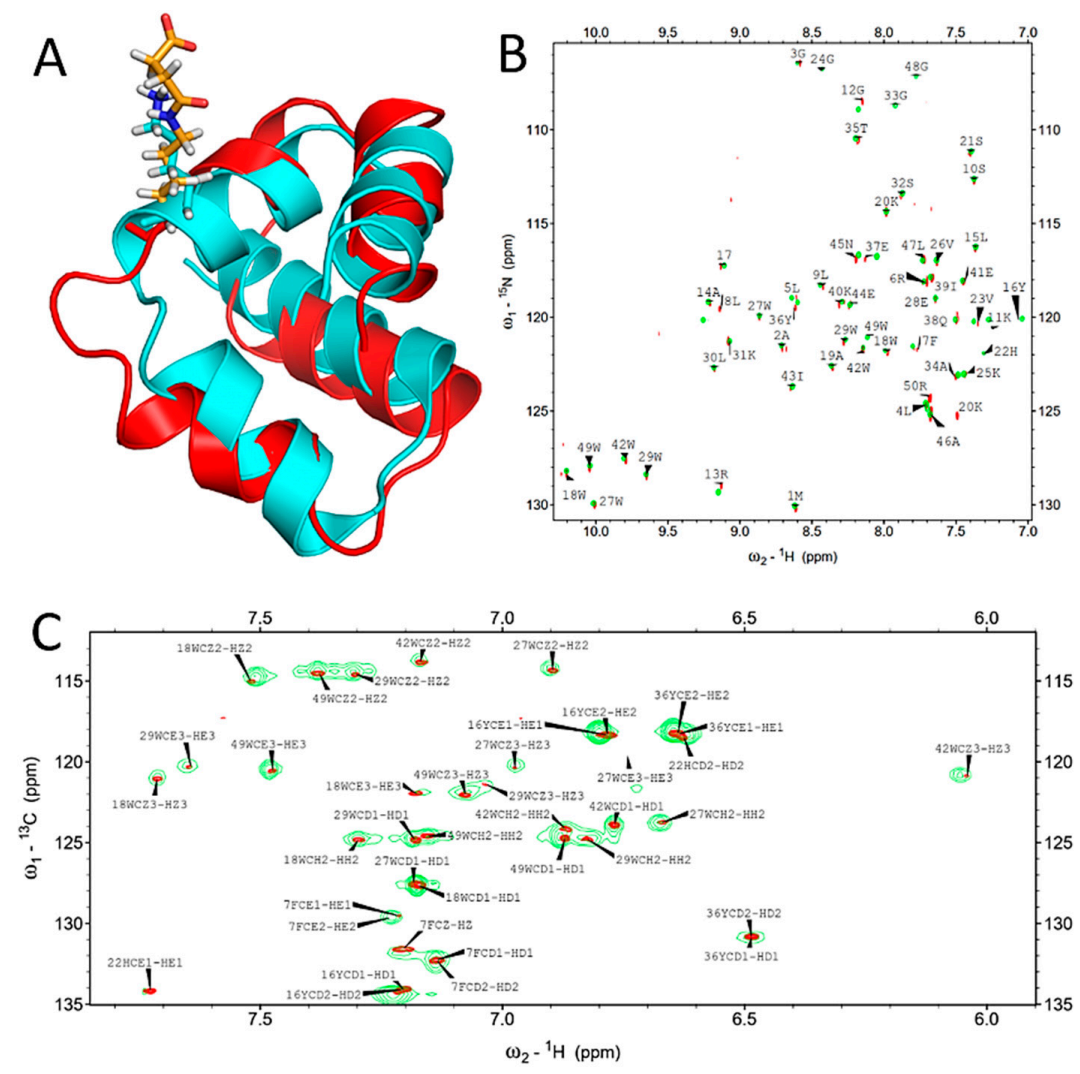

Figure 2. Results of NMR studies of the structure of unmodified and succinylated forms of BacSp222. Panel (A) illustrates the superimposition of the most representative structures of unmodified BacSp222 (cyan) and suc-K20-BacSp222 (red). The side chain of lysine 20 in both structures is represented as sticks-hydrogens white, nitrogens blue, carbons of unmodified lysine cyan, and carbons of modified lysine orange. The superimposition of ${ }^{1} \mathrm{H}_{-}{ }^{15} \mathrm{~N}$ HSQC spectra and ${ }^{1} \mathrm{H}_{-}{ }^{13} \mathrm{C}$ HSQC spectra tuned to aromatic carbons for unmodified BacSP222 (green) and suc-K20-BacSp222 (red) is presented in panels $(\mathbf{B}),(\mathbf{C})$, respectively.

The structure of succinylated BacSp222 calculated form NOEsy restraints was virtually identical to the structure of the unmodified peptide (Figure 2A). The mean global backbone 
RMSD calculated for the structures of modified and unmodified BacSp222 was $1.9 \pm 0.9 \AA$, indicating no significant structural differences. The statistics of 20 lowest energy structures of modified BacSp222 are presented in Supplementary Materials Table S1.

The comparison of the circular dichroism spectra recorded for BacSp222 and suc-K20BacSp222 indicated no significant changes. The calculated content of the helical structure within the succinylated form was slightly increased ( $75 \%$ in comparison to $81 \%$, Supplementary Materials Figure S2A), but this difference was within the relatively large uncertainty of such estimations. The $\mathrm{CD}$ analysis additionally allowed us to estimate the structural changes in the peptide molecule in the presence of liposomes, which was particularly interesting, as our previous studies revealed that the killing activity of BacSp222 is related to disruption of the bacterial membrane [21]. The spectra of native and succinylated bacteriocins in the presence and absence of liposomes are similar but, again, the calculated content of helical structures in suc-K20-BacSp222 was higher than in BacSp222 (94.4\% in comparison to $86.2 \%$; Supplementary Materials Figure S2A). It is also important that the near-UV $\mathrm{CD}$ spectroscopy, providing information on the tertiary structure, gave very similar shapes of the spectra (Supplementary Materials Figure S2B), suggesting that succinylation does not affect the overall shape of the bacteriocin molecule, which also confirms the results from NMR spectroscopy.

It can be speculated that the slight but consistent increase in the helical structure content in suc-K20-BacSp222 reflects a more rigid and/or compact structure of the unmodified bacteriocin. This conclusion is, however, not supported by our NMR result. Many authors emphasize that succinylation is an essential post-translational modification that alters e.g., the structure of proteins $[2,3,15,35]$; unfortunately, there are almost no experimental findings in favour of such a conclusion. Certain reports on in vitro succinylation of bovine serum albumin or glutamate dehydrogenase suggest such a possibility [4]; however, the only study evaluating the influence of physiological succinylation on the structure of a functional protein was focused on $\alpha \mathrm{B}$-crystallin from the human lens [20]. As shown by the results, the modification does not alter the secondary structure of crystallin but mildly perturbates its tertiary and quaternary structure, consequently leading to increased chaperone activity of this protein. Similarly, the influence of succinylation on the structure of BacSp222 in our case is negligible, if any.

\subsection{Succinylation Decreases the Antibacterial Activity of BacSp222 Bacteriocin}

The antibacterial activity of the BacSp222 forms against selected Gram-positive bacteria was compared with the use of the radial diffusion assay. The unmodified form inhibited the growth of each tested bacterial strain, as shown in our previous study [21]. The activity of the succinylated forms was significantly reduced, depending on the degree of succinylation and the bacterial strain. The residual activity of suc-K20-BacSp222 compared to the activity of the unmodified BacSp222 reached $93 \%$ against Micrococcus luteus, but only $3 \%$ against Staphylococcus intermedius. The residual bactericidal activity of suc-K11/K20BacSp222 against Lactococcus lactis was estimated at $49 \%$, while the peptide applied at the tested concentration was completely inactive against Staphylococcus aureus, Staphylococcus intermedius, or Staphylococcus pseudintermedius 22221 (unlike BacSp222) (Table 1). These results indicate that succinylation of lysine reduces the antibacterial activity of BacSp222. The observation is in agreement with other reports on succinylated bactericidal peptides. Cochrane and colleagues demonstrated reduced activity of analogues of the lipopeptide antibiotic cerexin with succinylated hydroxylysine residues [36]. Fuchs and others demonstrated an identical effect in the case of entianin bacteriocin succinylated at the tryptophan residue [37]. Unlike BacSp222, the succinylated forms had no antibacterial activity against producer strain Sp222. This allows us to speculate that succinylation provides a mechanism that protects producer cells against the autotoxicity of the secreted peptide. Other authors have suggested a similar functional role of peptides succinylation. For example, Bowers and colleagues suggested that lysine succinylation of thiazolyl peptides may serve a protective function for the producer organism [38]. 
Table 1. Antibacterial activities of post-translationally modified forms of BacSp222. The activity of suc-K20-BacSp222 and suc-K11/K20-BacSp222 against selected Gram-positive bacteria was assessed using the radial diffusion assay and expressed as percentages related to the activity of unmodified BacSp222.

\begin{tabular}{lcc}
\hline \multirow{2}{*}{ Bacteria } & \multicolumn{2}{c}{$\begin{array}{l}\text { Residual Antibacterial Activity in Relation to the } \\
\text { Activity of Unmodified BacSp222 (Percent } \pm \text { SD) }\end{array}$} \\
\cline { 2 - 3 } & Suc-K20-BacSp222 & Suc-K11/K20-BacSp222 \\
\hline Bacillus subtilis ATCC 6633 & $72.24 \pm 4.42$ & $32.66 \pm 6.78$ \\
Lactococcus lactis ŁOCK 0871 strain 239 & $80.32 \pm 3.67$ & $48.95 \pm 6.21$ \\
Micrococcus luteus ATCC 4698 & $92.97 \pm 0.56$ & $41.97 \pm 4.77$ \\
Staphylococcus aureus ATCC 25923 & $33.20 \pm 5.42$ & N.a. \\
Staphylococcus epidermidis ATCC 35547 & $64.19 \pm 7.80$ & $18.00 \pm 7.55$ \\
Staphylococcus intermedius ATCC 29663 & $3.24 \pm 2.51$ & N.a. \\
Staphylococcus pseudintermedius 222 & N.a. & N.a. \\
Staphylococcus pseudintermedius 22221 & $49.51 \pm 11.44$ & N.a. \\
\hline Na. & &
\end{tabular}
N.a.-not active.

\subsection{Succinylation limits BacSp222 Cytotoxic Activity Against Eukaryotic Cells}

BacSp222 is a multifunctional peptide with toxicity against both Gram-positive bacteria and eukaryotic cells [21]. Having demonstrated the reduced antibacterial activity of succinylated forms, we wondered whether a similar effect was exerted on cytotoxicity towards eucaryotic cells. The incubation of human neutrophils at $6 \mu \mathrm{M}$ BacSp222 forms for $2 \mathrm{~h}$ induced changes in the morphology of the cells, but most pronounced alterations were observed in cells treated with the unmodified BacSp222. The control (untreated) cells were spherical, whereas a significant number of BacSp222-treated cells were adherent, and their morphology was changed to bipolar. At the same time, only a minor fraction of the cells treated with the succinylated forms exhibited changes in their shape (Figure 3A). Neutrophils can change their shape rapidly, and this phenomenon is associated with migration to sites of inflammation and phagocytosis-dependent killing of microbes [39]. BacSp222 is known to have immunomodulatory properties [21] and such activity results from its interaction with eukaryotic cells. In turn, the immunomodulatory potential of succinylated forms remains to be determined.

We compared the cytotoxicity of the BacSp222 forms considered in this study. The viability of the cells was assessed by determining the level of intracellular ATP (neutrophils) or mitochondrial activity (other tested cells). Additionally, we assessed the integrity of cellular membranes with an LDH release assay. Significant differences between the activities of the BacSp222 forms were observed only at the highest tested concentration. At $3.13 \mu \mathrm{M}$, the viability of neutrophils treated with the unmodified peptide was around $50 \%$ in the assay conditions, whereas the viability of cells treated with suc-K20-BacSp222 or suc-K20-BacSp222 was about 95\% (Figure 3B,C). The reduction in the cytotoxic activity of the succinylated forms, compared to the unmodified bacteriocin, was also observed in the murine and human fibroblasts and in murine monocytic macrophages (Figure 4, Supplementary Materials Figure S3). Only the unmodified form of BacSp222 significantly affected the membrane integrity and viability of the RAW 264.7 and MDF cells (Figure 4). The extension of the incubation time from 4 to $24 \mathrm{~h}$ did not significantly affect the results (Supplementary Materials Figure S3). Overall, our results showing the lower cytotoxicity of succinylated forms of BacSp222 agree with literature reports on decreased activity of succinylated variants of other proteins. For example, a decrease in the cytotoxicity of succinylated forms of the EBI protease inhibitor against Molt4 cells was described by Ohba and colleagues [40]. We speculate that the reduced cytotoxic activity and diminished bactericidal effects of the succinylated forms of bacteriocin are a consequence of the modification of the overall charge of the peptide and charge distribution. Introduction of the negative charge is likely to lower the electrostatic affinity of the cationic peptide to negatively charged cell membranes. 
A

Control

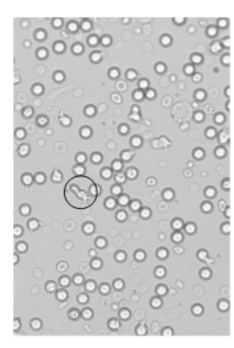

B

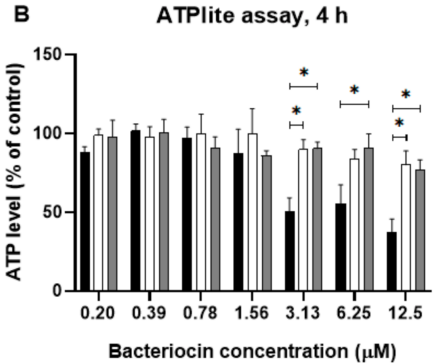

Suc-K20-BacSp222 Suc-K11/K20-BacSp222
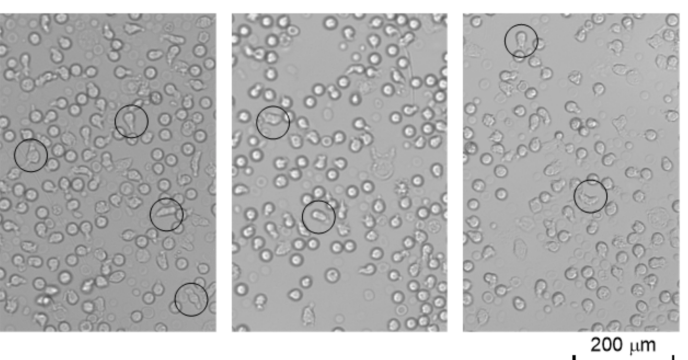

C LDH assay, $4 \mathrm{~h}$

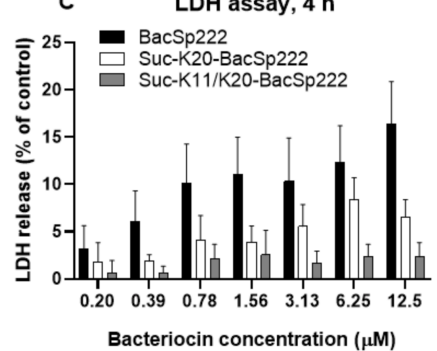

Figure 3. Comparison of the cytotoxic activity of different forms of bacteriocin BacSp222 against human neutrophils. The cells were isolated from healthy volunteers' blood and immediately exposed to various concentrations of BacSp222 forms for 4 h: unmodified BacSp222, suc-K20-BacSp222, or suc-K11/K20-BacSp222. After incubation, the cells were centrifuged, and the supernatants were transferred to a fresh plate for further analyses. (A) Morphological changes were analysed using an inverted light microscope. The circles indicate activated neutrophils. (B) The viability of the cells was determined using an ATPlite assay. (C) LDH activity in the medium indicates cell membrane damage in the presence of various forms of bacteriocin. ${ }^{*} p<0.05$.

RAW 264.7
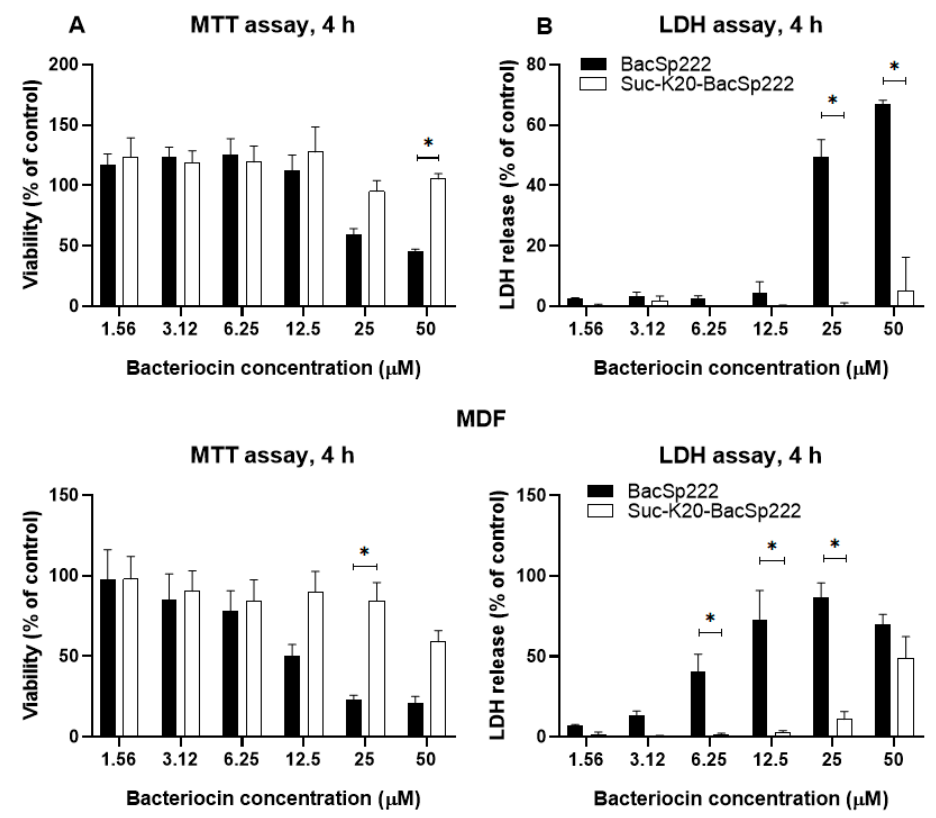

Figure 4. Cytotoxicity of different BacSp222 forms against murine monocyte-macrophage cells RAW 264.7 and murine primary dermal fibroblasts MDF. The cells were incubated for $4 \mathrm{~h}$ in a medium containing various concentrations of BacSp222 forms: unmodified BacSp222 and suc-K20-BacSp222. After incubation, the culture media were transferred on a fresh plate for further analyses. (A) The viability of the cells was measured using the MTT method. (B) The LDH activity in the medium indicates cell membrane damage in the presence of the various forms of bacteriocin. ${ }^{*} p<0.05$. 


\subsection{BacSp222 Succinylation Is a Nonenzymatic Process Dependent On Suc-CoA and $p H$, Whereas Desuccinylation Requires $\mathrm{NAD}^{+}$and Cytoplasmic Enzymes}

To establish whether BacSp222 succinylation may proceed via a nonenzymatic reaction, the unmodified bacteriocin was incubated with two physiological donors of the succinyl group existing in living bacteria-succinyl-CoA and succinate. Only succinyl-CoA was able to modify BacSp222 (Figure 5A). The reaction products were essentially identical to products excreted by living bacteria, i.e., suc-K20-BacSp222 and suc-K11/K20-BacSp222. The observed rate of the reaction was relatively fast: after $5 \mathrm{~min} \mathrm{13 \%}$ of bacteriocin was modified (Figure 5C). The reaction diminished gradually over time, and ca. 50\% succinylation level was achieved after $180 \mathrm{~min}$. No further increase of the succinylated forms was detected up to $300 \mathrm{~min}$ of the reaction. However, addition of a new dose of suc-CoA at 90 min of the reaction increased the level of succinylated forms to $60 \%$ (data not shown). These results indicate that the yield of in vitro BacSp222 succinylation is limited by the instability of suc-CoA in the water solution. This explanation is supported by literature data: suc-CoA solutions are stable for about $2 \mathrm{~h}$ [41]. The lysine modification reaction is not specific for BacSp222, as equivalent reactions have been described for a variety of proteins $[20,42,43]$; however, the site-specificity of modification is BacSp222 specific.
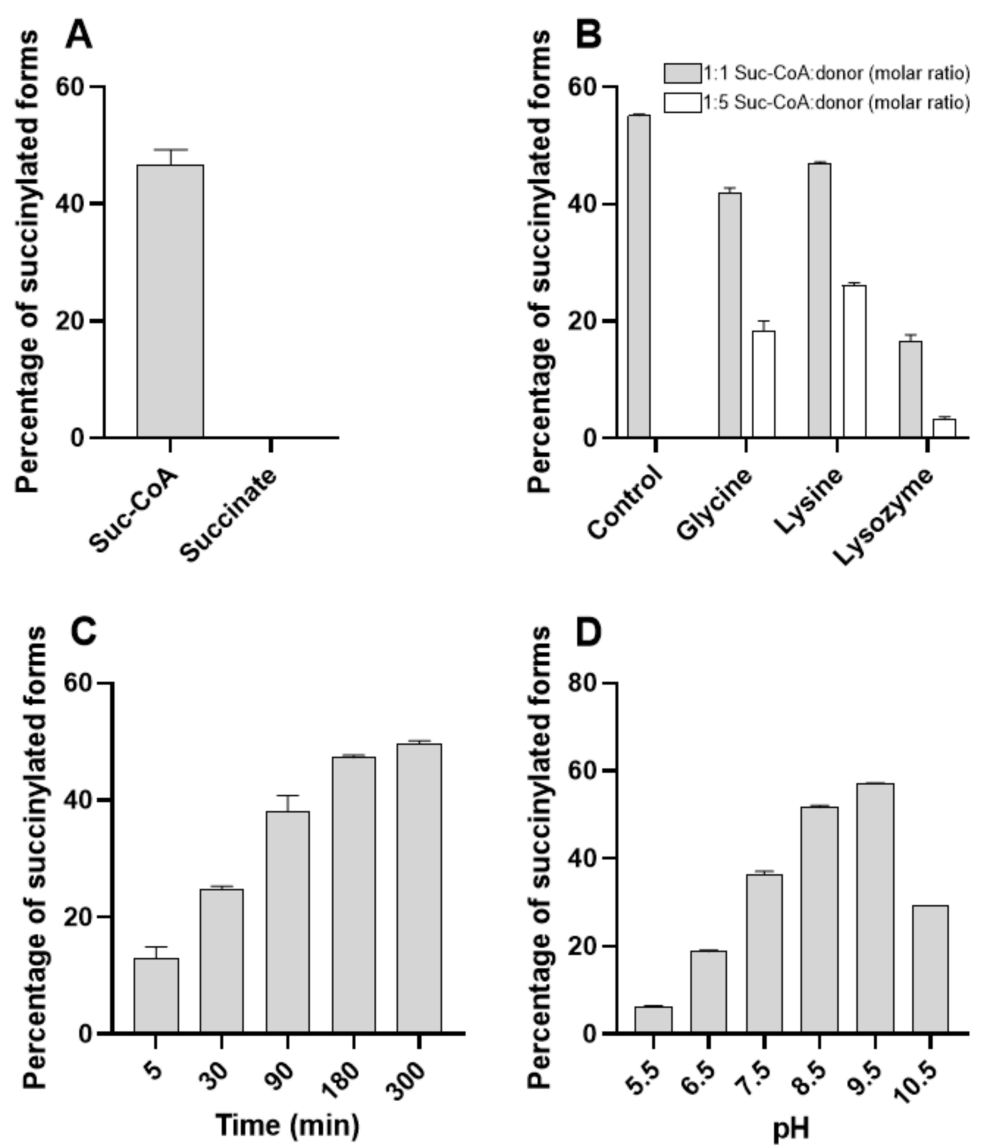

Figure 5. Results of in vitro studies of nonenzymatic succinylation of BacSp222. The unmodified form of BacSp222 was incubated with donors of the butanedioic group in different conditions. Next, the amount of BacSp222 forms was determined by RP-HPLC. (A) Ability of physiological donors of the butanedioic group to modify BacSp222. (B) Influence of the presence of other molecules containing amino groups on the modification of BacSp222 by suc-CoA. Effect of the reaction time (C) and $\mathrm{pH}$ of the solution (D) on the degree of BacSp222 succinylation.

The yield of succinylation of BacSp222 by suc-CoA was also dependent on the $\mathrm{pH}$ of the reaction. The highest succinylation rate was observed at $\mathrm{pH} 9.5$, whereas the modifica- 
tion of lysines in slightly acidic conditions ( $\mathrm{pH}$ 5.5) was practically blocked (Figure 5D). This observation agrees with the results of Wagner and Payne, who demonstrated that nonenzymatic BSA succinylation was most efficient at alkaline $\mathrm{pH}$ [43]. At $\mathrm{pH} 9.5, \mathrm{ly}-$ sine $\varepsilon$-amino groups are significantly protonated, as pKa of this moiety is 10.53 [44]. The most likely mechanism of succinylation comprises the nucleophilic attack of a positively charged $\varepsilon$-amino group of lysine on the carbonyl carbon of the succinyl group in suc$\mathrm{CoA}$. Additionally, alkaline $\mathrm{pH}$ increases the rate of formation of a highly reactive cyclic succinic anhydride intermediate, which reacts with primary amines in a dose-dependent manner [8].

The ability of suc-CoA to react with lysine residues is a common phenomenon [42,43]. Not surprisingly, our further experiments revealed that the excess of non-target amino groups reduces the efficiency of BacSp222 succinylation. For example, a 500-fold molar excess of lysozyme resulted in almost complete inhibition of the BacSp222 succinylation process, decreasing the level of succinylated forms from 55 to $3 \%$. Moreover, the presence of $\varepsilon$-amino groups and $\alpha$-amino groups affect the succinylation efficiency, as revealed during the reaction in the presence of free amino acids, glycine, and lysine (Figure 5B). Moreover, the presence of the bacterial intracellular or extracellular proteome inhi-bited the succinylation of BacSp222 (data not shown). These results suggest that succinylation of lysine by suc-CoA is nonspecific. The site of modification is most likely dictated by the structural arrangement of the peptide molecule.

We attempted to elucidate the mechanism of possible desuccinylation of BacSp222. Suc-K20-BacSp222 was incubated with bacterial cell lysates and/or with a NAD ${ }^{+}$solution, and the decrease in succinylated BacSp222 was determined. We showed that bacteriocin desuccinylation was catalysed by constituents of the intracellular proteome of Sp222 and a similar bacterial strain, Sp22221, only in the presence of NAD ${ }^{+}$(Supplementary Materials Figure S4). A common bacterial $\mathrm{NAD}^{+}$-dependent enzyme responsible for the lysine desuccinylation is the sirtuin-like protein CobB [17]. In fact, the Staphylococcus pseudintermedius genome contains $\mathrm{NAD}^{+}$-dependent protein deacylase [45].

Overall, our results indicate that, contrary to the succinylation process, BacSp222 desuccinylation requires enzymatic catalysis.

\subsection{Environmental Factors Affect the Level of Excreted Succinylated BacSp222 Forms}

The secretion of all forms of BacSp222 is growth phase-dependent. When bacteria were cultivated in the standard laboratory medium (TSB2), the highest concentrations were detected in the stationary growth phase, i.e., about $7 \mu \mathrm{M}$ for the unmodified BacSp222, ca. $3.5 \mu \mathrm{M}$ for suc-K20-BacSp222, and ca. $0.1 \mu \mathrm{M}$ for suc-K11/K20-BacSp222. The access to various nutrients affected the concentration of the BacSp222 forms produced by Sp222. In the nutrient-poor peptone water medium, the final concentrations of BacSp222 were about seven to five times lower (for the unmodified BacSp222 and suc-K20-BacSp222 forms, respectively), than in the high-nutrient media (Figure 6A). A similar correlation was described by Aasen and colleagues, who demonstrated that the level of sakacin P produced by Lactobacillus sakei depended on the availability of amino acids and growth factors [46]. The kinetics of production of different forms was unaffected by nutrient content.

Independently, we wanted to identify specific features that characterize Staphylococcus pseudintermedius 222 and ensure BacSp222 succinylation. To this end, we compared the cytosol $\mathrm{pH}$ value, the intracellular level of suc-CoA, and the degree of intracellular proteome succinylation between Sp222, two other representative strains of staphylococci (Staphylococcus pseudintermedius 22221 and Staphylococcus aureus ATCC 25923), and E. coli K12. No significant differences in the analysed factors were detected among the different bacteria. Staphylococcus aureus was characterized by the highest level of succinylated proteins, which correlated with the highest intracellular $\mathrm{pH}$. All staphylococci were characterized by similar concentrations of suc-CoA, while the Gram-negative bacterium was characterized by a five-fold lower concentration of this metabolite (Supplementary Materials Figure S6). Unfortunately, numerous attempts to produce BacSp222 in a heterologous expression system 
failed, so we were unable to determine whether the succinylation of the peptide is specific for S. pseudintermedius 222. However, the results obtained in this study indicate that the observed succinylation of excreted bacteriocin is not associated with the possible unusual biochemical features of Sp222 in relation to the other bacteria.
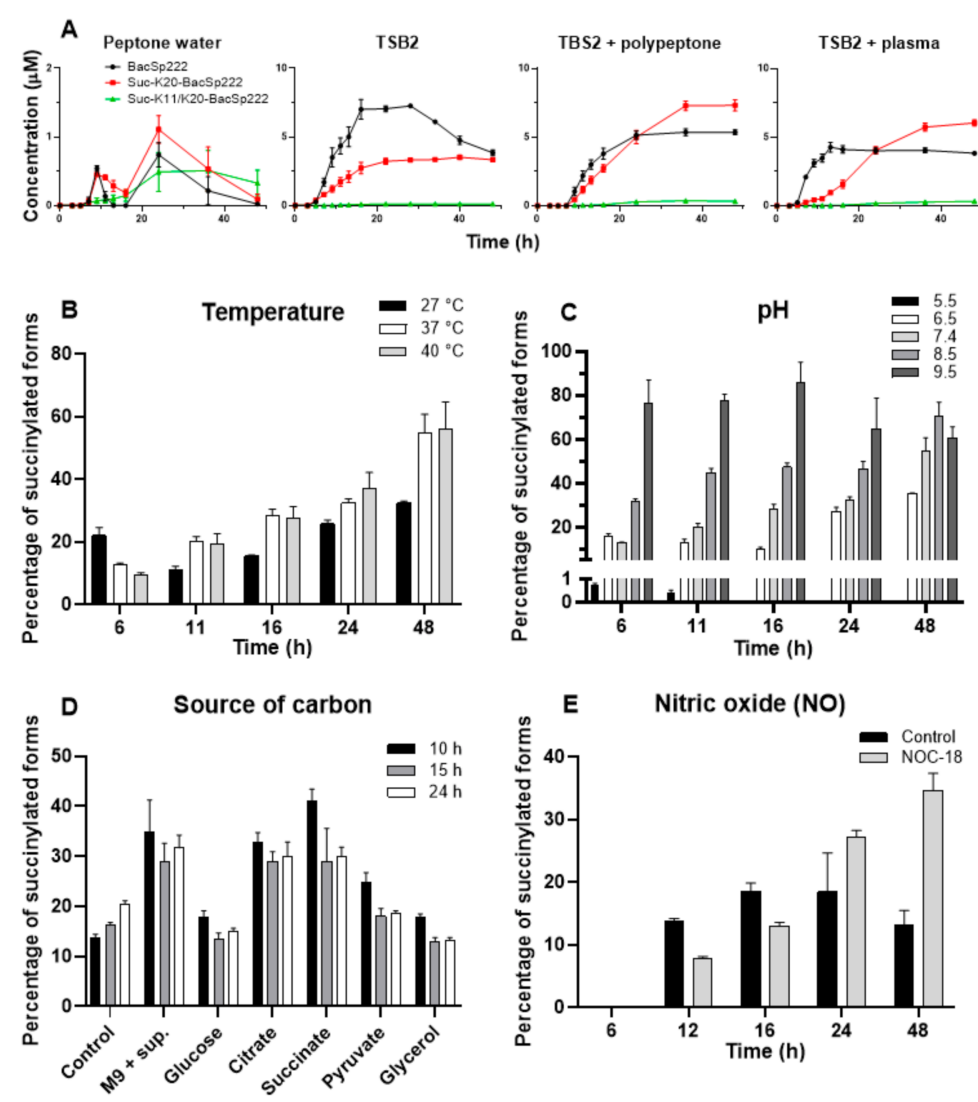

Figure 6. Effect of environmental factors on production of BacSp222 and its post-translationally modified forms by strain Sp222. The bacteria were cultured for various time intervals in different conditions; next, the levels of all BacSp222 forms were determined in the post-culture media by RPHPLC. (A) Effect of the access to nutrients on the biosynthesis of all BacSp222 forms. The successive panels illustrate the effect of culture temperature (B), $\mathrm{pH}$ of the culture (C), carbon source (D), and biological stress (presence of nitric oxide) (E) on the amount of modified BacSp222forms.

We evaluated the influence of the temperature and $\mathrm{pH}$ of the medium on succinylation of BacSp222 at different intervals of time during culture (Figure 6B). The lower temperature $\left(27^{\circ} \mathrm{C}\right)$ resulted in a lower percentage of succinylated forms of BacSp222, but this was related to the significantly lower metabolism and bacterial growth rate (two-fold reduction in the optical density of the culture, Supplementary Materials Figure S5B). A high correlation between $\mathrm{pH}$ and BacSp222 succinylation was observed. At $\mathrm{pH}$ 5.5, the modification of BacSp222 was entirely inhibited, while the overall amount of succinylated forms of BacSp222 at pH 9.5 was greater than $80 \%$ (Figure 6C). The observed increase in the succinylated forms at alkaline $\mathrm{pH}$ was probably not affected by changes in intracellular $\mathrm{pH}$, as bacteria can maintain the neutral $\mathrm{pH}$ of the cytosol even at extreme environmental $\mathrm{pH}$ values [47]. However, such maintenance of constant intracellular $\mathrm{pH}$ requires a different pattern of expressed proteins and significant changes in the entire metabolic pathways [48]. These overall modifications of cellular physiology are probably the cause of the observed increase in the degree of BacSp222 succinylation.

The level of suc-CoA depends on the activity of the Krebs cycle [49], while the activity of the Krebs cycle is modulated by the availability of particular carbon sources. We assessed whether the level of the BacSp222 forms depends on the type of the carbon source utilised 
during the bacterial culture. The BacSp222 forms were quantified upon the growth of Sp222 in media containing glucose, citrate, succinate, pyruvate, or glycerol as a primary carbon source and in a control medium without an additional source of carbon at different time points $(10,15$, and $24 \mathrm{~h}$ ) (Figure 6D). The citrate- or succinate-supplemented media were characterized by highest level of succinylated forms, while the lowest level was recorded in those containing glucose and pyruvate. Glucose is known to inhibit Krebs cycle enzymes and stimulate glycolysis. In turn, citrate and other TCA intermediate metabolites activate the Krebs cycle, while pyruvate and glycerol stimulate both glycolysis and the Krebs cycle [50]. We speculate that the high level of modified BacSp222 in samples containing TCA intermediates (citrate or succinate) may exactly be a consequence of the increased intracellular level of suc-CoA.

We have demonstrated that unmodified BacSp222 enhances interferon gammadependent NO production in murine macrophages. Nitric oxide is not only a multipotent immunomodulatory molecule but also an effective killing or stressing factor for bacterial cells [21]. We evaluated the effect of NO on the production of BacSp222 forms by Sp222 cells. We applied NOC-18 as a source of nitric oxide [51]. This compound has expected half-life in a solution of ca. $57 \mathrm{~h}$ and is able to release nitric oxide steadily. First, we determined that the presence of the bacteria reduced the half-life of NOC-18 (Supplementary Materials Figure S5F), but it was still sufficient for our purpose. The results of the experiment showed that the presence of $\mathrm{NO}$ in the bacterial culture enhanced the succinylation of BacSp222 in the late phase of the culture, and the levels of the succinylated forms were twice as high as in the control medium (Figure 6E). Nitric oxide has antibacterial activity [21]; however, the applied concentration of NOC-18 had no significant effect on the growth of bacteria-the growth was slightly diminished only in the initial phase of the culture (Supplementary Materials Figure S5E). Grosser and co-workers have demonstrated that NO induces the expression of nitric oxide resistance proteins in Staphylococcus aureus [52]. Possibly, the ability of Staphylococcus pseudintermedius to grow in the presence of $\mathrm{NO}$ is related to adequate changes in gene expression, which may further contribute to the enhanced level of BacSp222 succinylation. Nevertheless, such a conclusion requires further investigation. The present results indicate that the level of BacSp222 succinylation depends on the presence of a biological stressor, and Sp222 probably modulates the yield of post-translationally modified forms of BacSp222 in response to the host immune response. This phenomenon suggests that increased production of succinylated forms may be a mechanism to avoid the over-activation of the host immune system; however, this hypothesis requires further verification.

\section{Materials and Methods}

\subsection{Bacterial Strains and Culture Conditions}

The following bacterial strains were used in this study: Staphylococcus pseudintermedius 222/PCM 2791 (Sp222), Staphylococcus pseudintermedius LMG 22221 (Sp22221), Staphylococcus intermedius ATCC 29663, Staphylococcus aureus ATCC 25923 (Sa25923), Staphylococcus epidermidis ATCC 35547, Bacillus subtilis ATCC 6633, Lactococcus lactis ŁOCK 0871, Micrococcus luteus ATCC 4698, and Escherichia coli K12/ ATCC 10798 (EcK12). All bacteria were cultivated at $37^{\circ} \mathrm{C}$ in tryptic soy broth 2 (TSB2, Sigma, St. Louis, MO, USA), except E. coli, which was cultured in Luria-Bertani (LB) broth (Sigma, St. Louis, MO, USA).

\subsection{Eukaryotic Cells and Culture Conditions}

Human blood (containing citrate as an anticoagulant) from healthy volunteers was obtained from the Regional Centre of Blood Donation and Treatment (RCBDT) in Kraków, Poland. RCBDT deidentified the samples, which was relevant in order to keep the subjects identity confidential, and the study complied with appropriate rules of exclusion of human subjects. Neutrophils were isolated from the blood using centrifugation in a Ficoll-Paque Plus (GE Healthcare, Chicago, IL, USA) density gradient, and erythrocytes were eliminated by polyvinyl alcohol (POCH, Gliwice, Poland) treatment and osmotic lysis. Murine 
monocyte/macrophage RAW264.7 cells (ATCC TIB-71), murine monocyte/macrophage P388.D1 cells (ATCC CCL-46), and human skin fibroblast (HSF) cells (ATCC CRL-2522) were obtained from the American Type Culture Collection (Manassas, VA, USA). Murine primary dermal fibroblasts (MDF) were a kind gift from Dr. Krystyna Stalińska (Faculty of Biochemistry, Biophysics and Biotechnology, Jagiellonian University in Kraków). All cells were cultured at $5 \% \mathrm{CO}_{2}, 37^{\circ} \mathrm{C}$, and $>95 \%$ humidity in DMEM (GIBCO, Paislay, UK) containing $5 \%(v / v)$ foetal bovine serum (FBS, GIBCO, Paislay, UK) and 4.5 g glucose $/ \mathrm{L}$, except neutrophils, which were cultured in 10\% $(v / v)$ FBS in RPMI medium (LONZA, Basel, Switzerland).

\subsection{Isolation of BacSp222 and Its Succinylated Forms}

BacSp222 and its succinylated forms were purified from S. pseudintermedius 222 culture supernatant after $24 \mathrm{~h}$ of cultivation in TSB2 at $37{ }^{\circ} \mathrm{C}$ with shaking (200 rpm). After centrifugation $\left(5000 \times \mathrm{g}\right.$ for $15 \mathrm{~min}$ at $4{ }^{\circ} \mathrm{C}$ ), the supernatant was cooled to $4{ }^{\circ} \mathrm{C}$ and precipitated with ammonium sulphate to $60 \%$ saturation for four hours in an ice bath. The precipitated material was recovered by centrifugation at $21,000 \times \mathrm{g}$ for $30 \mathrm{~min}$ at $4{ }^{\circ} \mathrm{C}$. The pellet was dissolved in $\mathrm{H}_{2} \mathrm{O}$, acidified to $\mathrm{pH} 3.0$ with trifluoroacetic acid (TFA), centrifuged again, filtered through a $0.45 \mu \mathrm{m}$ filter, and subjected to reverse-phase high-pressure liquid chromatography (RP-HPLC) on a Discovery Bio Wide Pore C8 $250 \times 10 \mathrm{~mm}$ column (Sigma, St. Louis, MO, USA). The separations were performed at room temperature using an UltiMate 3000 HPLC system (Thermo, Waltham, MA, USA) and spectrophotometric detection at $220 \mathrm{~nm}$. Two solvents were used: A-0.1\% TFA $(v / v)$ in water and B$0.07 \%$ TFA in $80 \%$ acetonitrile (both $v / v$ ). After equilibration of the column at $60 \%$ of buffer B, a linear gradient from 60 to $100 \%$ of buffer B was applied over $20 \mathrm{~min}$ at a flow rate of $1.5 \mathrm{~mL} / \mathrm{min}$. Fractions containing BacSp222, suc-K20-BacSp222, and suc-K11/K20BacSp222 were collected, dried in a centrifugal evaporator, dissolved in water, and subjected to the second RP-HPLC step in identical conditions as above. The preparations of peptides obtained had a purity of over $99 \%$, as evaluated by analytical RP-HPLC chromatography. Each batch of purified bacteriocin was essentially free of lipopolysaccharide (LPS), as assayed by an E-TOXATE kit (Sigma, St. Louis, MO, USA).

\subsection{NMR Spectroscopy and Structural Calculations}

The nuclear magnetic resonance (NMR) spectra were acquired using a $0.5 \mathrm{mM}$ solution of native unlabelled suc-K20-BacSp222 dissolved in $650 \mu \mathrm{L}$ of an $\mathrm{H}_{2} \mathrm{O}: \mathrm{D}_{2} \mathrm{O}$ mixture (9:1, $v / v$ ) containing $100 \mathrm{mM}$ of deuterated sodium acetate, $\mathrm{pH}$ 5.0. The NMR experiments were performed using a Varian $700 \mathrm{MHz}$ DDR2 spectrometer equipped with an $\mathrm{HCN}$ probe at $25^{\circ} \mathrm{C}$ (calibrated with a standard ethylene glycol reference sample). The chemical shifts in the ${ }^{1} \mathrm{H}$ NMR spectra were reported with respect to external DSS-d4. The chemical shifts of the ${ }^{13} \mathrm{C}$ and ${ }^{15} \mathrm{~N}$ signals were referenced indirectly using frequency ratios of 0.251449530 for ${ }^{13} \mathrm{C} /{ }^{1} \mathrm{H}$ and 0.101329118 for ${ }^{15} \mathrm{~N} /{ }^{1} \mathrm{H}$ [53]. The assignment of the chemical shifts was based on our previous study of an unmodified BacSp222 peptide [22] and verified by analysis of a standard set of 2D NMR experiments consisting of TOCSY [54], NOESY [55], ${ }^{1} \mathrm{H}_{-}{ }^{15} \mathrm{~N}$ HSQC, and two ${ }^{1} \mathrm{H}^{13} \mathrm{C}$ HSQC [56] (the ${ }^{13} \mathrm{C}$ HSQC spectra were recorded with the offset, spectral widths, and ${ }^{13} \mathrm{C}-{ }^{1} \mathrm{H}$ coupling constant adjusted to aliphatic or aromatic carbons, respectively). The experimental data were processed using the NMRPipe software package [57], while the processed spectra were analysed with SPARKY software [58]. Relevant parameters of the NMR spectra are provided in Supplementary Materials Table S1. The ${ }^{1} \mathrm{H},{ }^{13} \mathrm{C}$, and ${ }^{15} \mathrm{~N}$ resonance assignments were deposited in BioMagResBank (BMRB) under accession code 34614 .

Distance constraints were derived from the 2D NOESY spectrum. Initial structural calculations were performed with CYANA 3.98 software [59], and the automatic NOESY assignment procedure provided 235 distance constraints. The additional restraints for the backbone $\varphi$ and $\psi$ angles for regions with a well-defined secondary structure (alpha-helices) were derived from the chemical shifts using the TALOS-N server [60]. XPLOR-NIH 2.26 
was used for structural calculations [61]. The topology of two additional amino acids (formylated methionine at the $\mathrm{N}$-terminus and succinylated lysine) was manually added to the XPLOR-NIH 2.26 topology file. Evaluation of the suc-K20-BacSp222 final structures was performed with the Protein Structure Validation Server (PSVS) [62]. Twenty structures with the lowest energy (out of the 400 calculated in the final structural refinement) were deposited in PDB under accession code 7NYI.

\subsection{Spectroscopy}

The circular dichroism (CD) spectra were obtained using 0.1 (for the $190-240 \mathrm{~nm}$ range) or 0.5 (for the $240-350 \mathrm{~nm}$ range) $\mathrm{mg} / \mathrm{mL}$ solutions of BacSp222 or suc-K20-BacSp222 dissolved in $50 \mathrm{mM}$ sodium phosphate $\mathrm{pH}$ 6.0. All measurements were made in a $1 \mathrm{~mm}$ quartz cuvette using a J-715 spectropolarimeter (Jasco, Tokyo, Japan) at $25^{\circ} \mathrm{C}$ without or in the presence of unilamellar dipalmitoylphosphatidyl glycerol (DPPG, Sigma, St. Louis, MO, USA) liposome suspension. The liposomes were prepared using an Avanti Polar Lipids syringe extruder equipped with a $100 \mathrm{~nm}$ filter and mixed with bacteriocins at a 100:1 phospholipid-to-bacteriocin molar ratio. All spectra were recorded with appropriate blank subtractions and averaged from three independent measurements. The amounts of structural motifs were calculated using the BeStSel server $[63,64]$.

\subsection{Biochemical Techniques}

The N-terminal amino acid sequences were determined using automatic protein sequencers Procise 491 (Applied Biosystems, Fosters City, CA, USA) and PPSQ-31A (Shimadzu, Kyoto, Japan). The concentration of purified bacteriocin and its succinylated forms was determined by amino acid analysis as described previously [65], while the concentration of these peptides in mixtures or biological fluids was determined by analytical RP-HPLC using a Discovery Bio Wide Pore C18 $250 \times 4.6 \mathrm{~mm}$ column (Sigma, St. Louis, $\mathrm{MO}$, USA) and a linear gradient $0-100 \%$ of buffer B for $15 \mathrm{~min}$ developed at $40{ }^{\circ} \mathrm{C}$. The other details of the HPLC separations were identical to those described in Section 3.3. The system was calibrated using a set of standard solutions of the bacteriocin and its succinylated forms.

Digestion of BacSp222 and its succinylated forms by trypsin (Gold grade, Pierce, Rockford, IL, USA) was performed for $17 \mathrm{~h}$ at $37^{\circ} \mathrm{C}$ in $0.2 \mathrm{M}$ Tris-HCl, $\mathrm{pH} 8.1$, containing $4 \%(w / v)$ sodium deoxycholate, using a 1:20 enzyme-to-substrate mass ratio. To denature the peptides and facilitate digestion, the reaction mixtures were heated to $99{ }^{\circ} \mathrm{C}$ for $5 \mathrm{~min}$ and chilled. Trypsin was added after chilling. The peptide fragments were purified by RPHPLC using a Discovery Bio Wide Pore C18 $4.6 \times 250 \mathrm{~mm}$ column (Sigma, St. Louis, MO, USA) and a linear gradient $0-100 \%$ of buffer B for 30 min developed at room temperature. The other details of the HPLC separations were identical to those described in Section 3.3.

A synthetic peptide SRVLDGLVMTTISSSK was used to obtain the peptide standard containing $\mathrm{N}-\varepsilon$-succinyl-lysine. A quantity of $10 \mathrm{nmol}$ of the peptide was dissolved in $1 \mathrm{~mL}$ of $0.2 \mathrm{M}$ sodium borate, $\mathrm{pH} 8.2$, and $3 \mathrm{mg}$ of solid succinic anhydride (Sigma) was added. The reaction was performed at room temperature for $1 \mathrm{~h}$ on a magnetic stirrer and at constant $\mathrm{pH}$ maintained by dropwise addition of a $5 \mathrm{M} \mathrm{NaOH}$ solution. After the reaction, the peptide was digested by cyanogen bromide to obtain the peptide fragment TTISSSL-suc-K, which was used to calibrate the automatic protein sequencer.

For mass spectrometry, the peptides were dissolved in $30 \%$ methanol with $0.1 \%$ formic acid (both $v / v$ ). An HTC Ultra ETD II mass spectrometer equipped with an electrospray ionization ion source and an electron-transfer dissociation II fragmentation module (Bruker) was used. The samples were injected directly using a syringe pump at a flow rate of $180 \mu \mathrm{L} /$ hour. The analyses were performed in a positive ion mode with a capillary voltage of $3.5 \mathrm{kV}$, a nebulizer pressure of $10 \mathrm{psi}$, a drying gas flow of $5 \mathrm{Lmin}$, and an ion source temperature of $300{ }^{\circ} \mathrm{C}$. The spectra were acquired in the MS and MS ${ }^{\mathrm{n}}$ modes in the range of 100-3000 m/ $z$ with both CID (collision-induced dissociation) and ETD (electron-transfer 
dissociation) ion fragmentation. The tandem MS data were processed manually using DataAnalysisTM 4.0 and BiotoolsTM 3.2 software (Bruker, Billerica, MA, USA).

To determine the physicochemical character of the excreted forms of bacteriocin, $10 \mathrm{~mL}$ of the bacterial post-culture medium was prepared by centrifugation at $10,000 \times g$ for $20 \mathrm{~min}$ to remove bacterial cells. The supernatant was fractionated using a 100-kDa cut-off membrane (Millipore, Burlington, MA, USA). The filtrate and the supernatant containing possible macromolecular aggregates or membrane vesicles excreted by bacterial cells were solubilized with $6 \mathrm{M}$ guanidine hydrochloride (Sigma). The level of each form of bacteriocin was determined by RP-HPLC using a Discovery Bio Wide Pore C8 $250 \times 4.6 \mathrm{~mm}$ column (Sigma) developed in a linear gradient from 0 to $100 \%$ of buffer B over $15 \mathrm{~min}$ at $40{ }^{\circ} \mathrm{C}$ and a flow rate of $1 \mathrm{~mL} / \mathrm{min}$. The other details of the separations were identical to those described in Section 3.3.

Bacterial cell lysate was obtained via a combination of enzymatic and mechanical factors. A volume of $10 \mathrm{~mL}$ of bacterial culture was centrifuged at $5000 \times \mathrm{g}$ for $10 \mathrm{~min}$. The pellet was washed 3 times wth PBS and suspended in $80 \mu \mathrm{L}$ of phosphate-buffered saline (PBS, Sigma). The cells were pretreated with $40 \mu \mathrm{g} / \mathrm{mL}$ lysostaphin (Sigma) and $125 \mathrm{U} / \mathrm{mL}$ Omni nuclease (Eurx, Gdańsk, Poland) with shaking at 1,000 rpm for $1 \mathrm{~h}$ at $37^{\circ} \mathrm{C}$. The suspension was sonicated using a UP50H sonicator (Hielscher, Teltov, Germany, 30 pulses at $100 \%$ of power, amplitude 0.5 ). The incubation and sonication steps were repeated. As a final step, the suspension was centrifuged at $15,000 \times g$ for $20 \mathrm{~min}$ at $4{ }^{\circ} \mathrm{C}$. The collected lysate was stored at $-20^{\circ} \mathrm{C}$.

The concentration of succinyl-coenzyme A (suc-CoA) was determined by RP-HPLC using a Discovery C18 $250 \times 4.6 \mathrm{~mm}$ column (Sigma). Two solvents were used: A- $150 \mathrm{mM}$ $\mathrm{NaH}_{2} \mathrm{PO}_{4}$ containing $5 \%$ methanol $(v / v)$ and $\mathrm{B}-150 \mathrm{mM} \mathrm{NaH}_{2} \mathrm{PO}_{4}$ containing $30 \%$ methanol $(v / v)$. After equilibration of the column at $0 \%$ of buffer $\mathrm{B}$, a linear gradient from 0 to $100 \%$ of buffer B was applied over $7.5 \mathrm{~min}$. The flow rate was $1 \mathrm{~mL} / \mathrm{min}$. The spectrophotometric detection was performed at $254 \mathrm{~nm}$ while the temperature of the column was maintained at $40{ }^{\circ} \mathrm{C}$. Before measurements, the column was calibrated using a set of standard solutions of suc-CoA (Santa Cruz Biotechnology, Santa Cruz, CA, USA).

The level of lysine succinylation in intracellular bacterial proteomes was determined by Western Blotting. Tris-glycine sodium dodecyl sulphate polyacrylamide gel electrophoresis (SDS-PAGE) was performed in reducing conditions using Mini-Protean Precast Gels (BioRad, Hercules, CA, USA). After electrophoresis, the proteins were transferred on a $0.4 \mu \mathrm{m}$ pore size polyvinylidene difluoride (PVDF) membrane (Millipore, Burlington, MA, USA) using $10 \mathrm{mM} \mathrm{N}$-cyclohexyl-3-aminopropanesulfonic acid (CAPS) buffer, $\mathrm{pH}$ 11.0, containing $10 \%(v / v)$ methanol. The transfer was evaluated by transient staining the membranes using $0.5 \%(w / v)$ Ponceau S dissolved in 5\% $(v / v)$ acetic acid. The immunodetection was performed using a $0.05 \%(v / v)$ solution of anti-succinyl lysine (anti-Ksu) rabbit polyclonal antibodies (PTM BIO, Cesarea, Israel) dissolved in 5\% $(w / v)$ skimmed milk in Tris-buffered saline containing $0.05 \%$ Tween-20 (TBST). The primary antibodies were detected using a $0.1 \%(v / v)$ solution of HRP-labelled goat anti-rabbit IgG antibodies (Sigma, St. Louis, MO, USA) in $5 \%(w / v)$ skimmed milk in TBST. DAB peroxidase substrate (Sigma) was used to visualize the results.

Bioinformatic prediction of succinylation sites in BacSp222 was performed using available algorithms: SuccinSite [32], GPSuc [31], iSuc-Opt predictor [30], pSuc-Lys predictor [29], iSucPseAAC [34], and SucFind [28].

\subsection{Antibacterial Radial Diffusion Assay}

A radial diffusion assay was used to compare the bactericidal activity of the BacSp222 forms. Soft TSB plates $(0.75 \%$ agarose, $w / v)$ contained the tested bacteria. Aliquots of $5 \mu \mathrm{L}$ of the tested bacteriocin were added as drops at different sites. BacSp222 was applied in serial two-fold dilutions at concentrations ranging from $0.62 \mu \mathrm{M}$ to $40 \mu \mathrm{M}$, whereas both succinylated forms were used only at the highest concentration $(40 \mu \mathrm{M})$. After $16 \mathrm{~h}$ of incubation at $37^{\circ} \mathrm{C}$, the antibacterial activity of the peptides was determined as the diameter 
of the bacterial growth inhibition zone. Because the correlation between the diameter of inhibition zones and concentration of the peptides was nonlinear the antibacterial activities of both modified forms of the bacteriocin were calculated and presented as a percentage of the activity of unmodified BacSp222.

\subsection{Analysis of the Effect of Bacteriocin on Eukaryotic Cells}

Human blood neutrophils were transferred to a half-area 96 -well plate $(50,000$ cells per well in $50 \mu \mathrm{L}$ RPMI, 10\% FBS, Sigma, St. Louis, MO, USA). $5 \mu \mathrm{L}$ of PBS or bacteriocin solutions was added and the cells were cultured for $4 \mathrm{~h}$. After incubation, the cells were centrifuged at $1280 \times g$, and the supernatants were used to determine the activity of lactate dehydrogenase (LDH). Cell viability was determined using an ATPlite kit. Both assays were performed in accordance with the manufacturers' instructions (Roche, Basel, Switzerland, and Perkin Elmer, Waltham, MA, USA, respectively). A positive control for the LDH release was obtained by treating the cells with 5\% (v/v) Triton X-100 (Sigma, St. Louis, MO, USA) in RPMI. The absorbance (in the case of LDH detection) and luminescence (for the viability assay) were measured with the use of a Synergy H1 hybrid plate reader controlled by Gene5 version 2.00.18 Software (BIOTEK Instruments, Winooski, VT, USA). The viability of cells was calculated using the following formula:

$$
\text { ATP level }\left(\% \text { of control) }=\frac{\text { Luminescence of bacteriocin-treated cells }}{\text { Luminescence of PBS-treated cells }} \times 100 \%\right.
$$

LDH activity was calculated according to the formula:

$$
\text { LDH release }(\% \text { of control })=\frac{\text { Absorbance of bacteriocin-treated cells }}{\text { Absorbance of Triton X-100-treated cells }} \times 100 \%
$$

The morphology of neutrophils upon the treatment with bacteriocins was inspected using an inverted light microscope DM IL LED Fluo (Leica, Wetzlar, Germany).

RAW264.7, MDF, P388.D1, and HSF cells were seeded on half-area 96-well plates in $50 \mu \mathrm{L}$ of growth medium at a density of 20,000 cells per well. After $24 \mathrm{~h}$, the media were removed and replaced with fresh DMEM containing $2 \%$ FBS and $5 \mu \mathrm{L}$ of PBS (for control cells) or $5 \mu \mathrm{L}$ of bacteriocins (final concentrations: 50, 25, 12.5, 6.25, 3.12, and 1.56 $\mu \mathrm{M}$ ). After 4- or 24-h exposure to the bacteriocins, the supernatants were collected and used to measure LDH activity (as described above), while the viability of the cells was determined by an MTT assay according to the standard protocol. All measurements were performed using a Synergy H1 hybrid reader running with Gene5 v2.00.18 Software (BIOTEK Instruments, Winooski, VT, USA). The viability of cells was calculated using the following formula:

$$
\text { Viability }(\% \text { of control })=\frac{\text { Absorbance of bacteriocin-treated cells }}{\text { Absorbance of PBS-treated cells }} \times 100 \%
$$

\subsection{In Vitro Analysis of Succinylation and Desuccinylation of BacSp222}

BacSp222 or suc-K20-BacSp222 were incubated at $37{ }^{\circ} \mathrm{C}$ for $3 \mathrm{~h}$ (unless otherwise indicated) in PBS or in Britton-Robinson buffer with a 3-carboxypropanoyl group donor such as succinyl coenzyme A sodium salt (Santa Cruz Biotechnology) or disodium succinate (Sigma) at molar ratios of 1:100 (for succinylation studies) or independently with the bacterial lysate or/and with $25 \mathrm{mM} \mathrm{NAD}^{+}$(Roanal) (in case of desuccinylation studies). The $\mathrm{pH}$ and ionic strength of the Britton-Robinson buffer were adjusted by $\mathrm{KOH}$ and $\mathrm{NaCl}$, respectively. Compounds containing amino groups i.e., glycine (Sigma), lysine (Sigma, St. Louis, MO, USA), and human lysozyme (Sigma, St. Louis, MO, USA) and the BacSp222 variants were used at compound:donor molar ratios of 1:100 or 1:500. The concentration of the BacSp222 forms was determined by RP-HPLC using a Discovery Bio Wide Pore C8 $250 \times 4.6 \mathrm{~mm}$ column (Sigma, St. Louis, MO, USA) and a linear gradient from 0 to $100 \%$ of buffer B over 15 min developed at $40{ }^{\circ} \mathrm{C}$ and a flow rate of $1 \mathrm{~mL} / \mathrm{min}$. The other details of the separations were identical to those described in Section 3.3. The results are 
presented as a percentage of the succinylated form in the pool of all BacSp222 forms (for succinylation studies) or a percentage of detected suc-K20-BacSp222 relative to the control suc-K20-BacSp222 sample (in the desuccinylation studies).

\subsection{Analysis of the Influence of Environmental Factors on the Level of Succinylated Forms of BacSp222}

Staphylococcus pseudintermedius 222 (Sp222) was cultivated in TSB2 or in M9 medium with supplementation dedicated to staphylococci [66] at $37^{\circ} \mathrm{C}$ (unless otherwise stated) and $180 \mathrm{rpm}$ shaking for different periods. $\mathrm{pH}$ of the media were adjusted with $\mathrm{NaOH}$ or $\mathrm{HCl}$. The carbon sources included glucose (BioShop, Burlington, ON, Canada), citrate $(\mathrm{POCH})$, succinate (Merck), pyruvate (Eurochem BGD), or glycerol (BioShop, Burlington, ON, Canada), and were tested at a $60 \mathrm{mM}$ concentration. The donor of nitric oxide, NOC-18 (ChemCruz, Santa Cruz, CA, USA), was used at a $4 \mu \mathrm{M}$ concentration. Optical density (OD) at $600 \mathrm{~nm}$ was used to monitor the growth. After cultivation, the bacterial suspension was centrifuged at $10,000 \times g$ for 5 min to remove the cells, and the concentration of all BacSp222 forms was determined by RP-HPLC (in conditions as described in Section 3.9). The results were shown as a percentage of succinylated forms in relation to the amount of all BacSp222 forms.

Nitric oxide release was determined using the Griess assay. Briefly, $50 \mu \mathrm{L}$ of $1 \%(w / v)$ sulfanilic acid (Sigma, St. Louis, MO, USA) in 2.5\% (v/v) $\mathrm{H}_{3} \mathrm{PO}_{4}$ and $50 \mu \mathrm{L}$ of $0.1 \%(w / v)$ $\mathrm{N}$-(1-naphtyl) ethylenediamine dihydrochloride (Sigma) in 2.5\% $(v / v) \mathrm{H}_{3} \mathrm{PO}_{4}$ were added to $100 \mu \mathrm{L}$ of post-culture medium. Absorbance was determined at $545 \mathrm{~nm}$ using a Synergy H1 hybrid reader and Gene5 v2.00.18 Software (BIOTEK Instruments, Winooski, VT, USA).

\subsection{Determination of Intracellular $p H$ in Bacteria}

The bacteria were incubated for $30 \mathrm{~min}$ at $37^{\circ} \mathrm{C}$ with shaking (300 rpm) upon addition of $10 \mu \mathrm{g} / \mathrm{mL}$ of the $\mathrm{pH}$-sensitive dye BCECF-AM (Thermo, Waltham, MA, USA). The cultures were centrifuged for $5 \mathrm{~min}$ at $5000 \times g$, and the bacterial pellet was suspended at OD of 0.4 in PBS supplemented with $25 \mathrm{mM}$ glucose. The suspensions were mixed with PBS (control sample) or with a solution of Intracellular pH Calibration Buffer (Thermo) containing $10 \mu \mathrm{M}$ nigericin (Thermo, Waltham, MA, USA) and $10 \mu \mathrm{M}$ valinomycin (Thermo, Waltham, MA, USA) (for Gram-positive bacteria) or $10 \mu \mathrm{M}$ CCCP (Sigma, St. Louis, MO, USA) (in the case of Gram-negative bacteria) at a volume ratio 1:1. The mixtures were incubated in darkness at room temperature for $45 \mathrm{~min}$, and the fluorescence at excitation/emission wavelengths of $440 / 530 \mathrm{~nm}$ and 505/530 nm (for Gram-positive bacteria) or $490 / 530 \mathrm{~nm}$ (in the case of Gram-negative bacteria) was determined using a Synergy H1 hybrid reader and with Gene5 version 2.00.18 Software (BIOTEK Instruments, Winooski, VT, USA). The results were analysed using online tool “Quest Graph ${ }^{\mathrm{TM}}$ Four Parameter Logistic (4PL) Curve Calculator" [67].

\subsection{Statistics}

Statistical significance for the cytotoxicity assays was calculated by one-way ANOVA on ranks using Statistica v13.3 software (StatSoft), with $p<0.05$ considered significant. The bars on the charts represent the average values from three experiments \pm SD.

\section{Conclusions}

We have shown that S. pseudintermedius 222 excrete bacteriocin BacSp222 in three forms: unmodified and two forms (suc-K20-BacSp222 and suc-K11/K20-BacSp222) modified posttranslationally by succinyl groups attached to $\varepsilon$-amino moieties of lysine residues. The NMR and CD experiments have revealed that the structure of suc-K20-BacSp222 does not significantly differ from that of the native unmodified bacteriocin. At the same time, succinylation of BacSp222 leads to a significant reduction in antibacterial and cytotoxic activity. We have also shown that succinylation is a nonenzymatic process with the highest efficiency at slightly alkaline $\mathrm{pH}$, and the Krebs cycle intermediate succinyl-CoA is the most likely physiological donor capable of modifying the bacteriocin. The amount of BacSp222 
excreted by bacteria depends on various environmental factors. We demonstrated that the level of BacSp222 forms was associated with access to nutrients and reached the highest values in high-nutrient media. Moreover, the amount of the excreted succinylated forms was higher at a temperature close to that of the host body and in the post-culture media supplemented with citrate or succinate than in those supplemented with glucose. Furthermore, a higher level of succinylated forms of the peptide was excreted when Sp222 was cultured in stress conditions (nitric oxide).

Overall, the reduced bactericidal activity of succinylated forms of bacteriocin interpreted together with the increased amount of succinylated forms in stress conditions suggest that post-translational modifications are a form of protection of producer cells against the secreted bacteriocin. We speculate that (i) the reduced cytotoxic activity of the succinylated forms of the bacteriocin, (ii) the stimulatory effect of NO on succinylation, and (iii) the fact that Sp222 is an opportunistic specie may indicate that the posttranslational modification of BacSp222 is an element of the mechanism responsible for making Sp222 a natural element of the canine bacterial flora rather than a conventional pathogen.

Supplementary Materials: The following are available online at https://www.mdpi.com/article/10

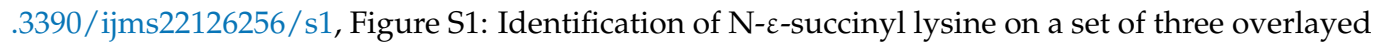
chromatograms from sequencing cycles of tryptic peptide fragments obtained from succinylated forms of BacSp222; Figure S2: Results of CD measurements in the far (A) and near (B) UV region for unmodified and succinylated forms of BacSp222 recorded both without and in the presence of DPPG liposomes; Figure S3: Comparison of the cytotoxic activity of different forms of bacteriocin BacSp222 against murine monocyte-macrophage cells (RAW 264.7), murine dermal primary fibroblasts (MDF), human skin primary fibroblasts (HSF), and murine monocyte-macrophage cells (P388.D1); Figure S4: Results of in vitro studies of desuccinylation of suc-K20-BacSp222; Figure S5: Growth curves of Sp222 bacteria showing the effect of (A) access to nutrients, (B) temperature of bacterial culture, (C) $\mathrm{pH}$ value of the medium, (D) source of carbon, (E) presence of nitric oxide; Figure S6: Comparison of selected biochemical factors between Staphylococcus pseudintermedius 222 and the other bacteria, i.e., Staphylococcus pseudintermedius 22221, Staphylococcus aureus 25923, and Escherichia coli K12; Table S1: NMR-derived constraints and statistics for the suc-K20-BacSp222 structure calculated with XPLOR-NIH 2.26.

Author Contributions: Conceptualization, P.M.; methodology, P.M., J.Ś., M.N. and M.B.; software, J.Ś. and M.N.; validation, P.M., J.Ś., M.N. and M.B.; formal analysis, P.M.; investigation, P.M., J.Ś., M.N., M.B., O.B. and A.W.; resources, P.M.; data curation, P.M.; writing-original draft preparation, P.M., J.Ś., M.N., M.B. and O.B.; writing—review and editing, P.M., J.Ś., G.D. and A.K.; visualization, P.M. and J.S.; supervision, P.M.; project administration, P.M.; funding acquisition, P.M. All authors have read and agreed to the published version of the manuscript.

Funding: The work was financed by National Science Centre, Poland, in the framework of the project No 2018/31/B/NZ3/01226 (to P.M.).

Institutional Review Board Statement: Not applicable.

Informed Consent Statement: Not applicable.

Data Availability Statement: The study did not report any data.

Acknowledgments: The authors thank Kinga Chlebicka, Łukasz Mazurek, Anna Mądry, Marcin Piejko and Benedykt Władyka for their precious tips and help. Krystyna Stalińska is acknowledged for providing us murine primary dermal fibroblasts.

Conflicts of Interest: The authors declare no conflict of interest.

\section{References}

1. Zhang, Z.; Tan, M.; Xie, Z.; Dai, L.; Chen, Y.; Zhao, Y. Identification of lysine succinylation as a new post-translational modification. Nat. Chem. Biol. 2011, 7, 58-63. [CrossRef] [PubMed]

2. Alleyn, M.; Breitzig, M.; Lockey, R.; Kolliputi, N. The dawn of succinylation: A posttranslational modification. Am. J. Physiol. Physiol. 2018, 314, C228-C232. [CrossRef] [PubMed] 
3. Sreedhar, A.; Wiese, E.K.; Hitosugi, T. Enzymatic and metabolic regulation of lysine succinylation. Genes Dis. 2020 , 7, 166-171. [CrossRef] [PubMed]

4. Weinert, B.T.; Schölz, C.; Wagner, S.A.; Iesmantavicius, V.; Su, D.; Daniel, J.A.; Choudhary, C. Lysine succinylation is a frequently occurring modification in prokaryotes and eukaryotes and extensively overlaps with acetylation. Cell Rep. 2013, 4, 842-851. [CrossRef] [PubMed]

5. Song, Y.; Wang, J.; Cheng, Z.; Gao, P.; Sun, J.; Chen, X.; Chen, C.; Wang, Y.; Wang, Z. Quantitative global proteome and lysine succinylome analyses provide insights into metabolic regulation and lymph node metastasis in gastric cancer. Sci. Rep. 2017, 7, 42053. [CrossRef]

6. Li, X.; Hu, X.; Wan, Y.; Xie, G.; Li, X.; Chen, D.; Cheng, Z.; Yi, X.; Liang, S.; Tan, F. Systematic identification of the lysine succinylation in the protozoan parasite Toxoplasma gondii. J. Proteome Res. 2014, 13, 6087-6095. [CrossRef]

7. Yang, Y.; Gibson, G.E. Succinylation links metabolism to protein functions. Neurochem. Res. 2019, 44, 2346-2359. [CrossRef]

8. Wagner, G.R.; Bhatt, D.P.; O'Connell, T.M.; Thompson, J.W.; Dubois, L.G.; Backos, D.S.; Yang, H.; Mitchell, G.A.; Ilkayeva, O.R.; Stevens, R.D.; et al. A class of reactive acyl-coa species reveals the non-enzymatic origins of protein acylation. Cell Metab. 2017, 25, 823-837. [CrossRef]

9. Gläser, L.; Kuhl, M.; Jovanovic, S.; Fritz, M.; Vögeli, B.; Erb, T.J.; Becker, J.; Wittmann, C. A common approach for absolute quantification of short chain CoA thioesters in prokaryotic and eukaryotic microbes. Microb. Cell Factories 2020, 19. [CrossRef]

10. Park, J.; Chen, Y.; Tishkoff, D.X.; Peng, C.; Tan, M.; Dai, L.; Xie, Z.; Zhang, Y.; Zwaans, B.M.M.; Skinner, M.E.; et al. SIRT5-mediated lysine desuccinylation impacts diverse metabolic pathways. Mol. Cell 2013, 50, 919-930. [CrossRef]

11. Zhang, K.; Xiong, Y.; Sun, W.; Wang, G.; Liu, W. Global proteomic analysis reveals widespread lysine succinylation in rice seedlings. Int. J. Mol. Sci. 2019, 20, 5911. [CrossRef]

12. Xie, L.; Liu, W.; Li, Q.; Chen, S.; Xu, M.; Huang, Q.; Zeng, J.; Zhou, M.; Xie, J. First succinyl-proteome profiling of extensively drug-resistant mycobacterium tuberculosis revealed involvement of succinylation in cellular physiology. J. Proteome Res. 2015, 14, 107-119. [CrossRef]

13. Zhou, H.; Finkemeier, I.; Guan, W.; Tossounian, M.A.; Wei, B.; Young, D.; Huang, J.; Messens, J.; Yang, X.; Zhu, J.; et al. Oxidative stress-triggered interactions between the succinyl- and acetyl-proteomes of rice leaves. Plant Cell Environ. 2018, 41, 1139-1153. [CrossRef]

14. Yang, M.; Wang, Y.; Chen, Y.; Cheng, Z.; Gu, J.; Deng, J.; Bi, L.; Chen, C.; Mo, R.; Wang, X.; et al. Succinylome analysis reveals the involvement of lysine succinylation in metabolism in pathogenic mycobacterium tuberculosis. Mol. Cell. Proteom. 2015, 14, 796-811. [CrossRef]

15. Zhao, Y.; Han, Y.; Sun, Y.; Wei, Z.; Chen, J.; Niu, X.; An, Q.; Zhang, L.; Qi, R.; Gao, X. Comprehensive succinylome profiling reveals the pivotal role of lysine succinylation in energy metabolism and quorum sensing of Staphylococcus epidermidis. Front. Microbiol. 2021, 11, 632367. [CrossRef]

16. Xie, Z.; Dai, J.; Dai, L.; Tan, M.; Cheng, Z.; Wu, Y.; Boeke, J.D.; Zhao, Y. Lysine succinylation and lysine malonylation in histones. Mol. Cell. Proteom. 2012, 11, 100-107. [CrossRef]

17. Colak, G.; Xie, Z.; Zhu, A.Y.; Dai, L.; Lu, Z.; Zhang, Y.; Wan, X.; Chen, Y.; Cha, Y.H.; Lin, H.; et al. Identification of lysine succinylation substrates and the succinylation regulatory enzyme CobB in Escherichia coli. Mol. Cell. Proteom. 2013, 12, 3509-3520. [CrossRef]

18. Kidwai, S.A.; Ansari, A.A.; Salahuddin, A. Effect of succinylation (3 carboxypropionylation) on the conformation and immunological activity of ovalbumin. Biochem. J. 1976, 155, 171-180. [CrossRef]

19. Habeeb, A.F.S.A. Quantitation of conformational changes on chemical modification of proteins: Use of succinylated proteins as a model. Arch. Biochem. Biophys. 1967, 121, 652-664. [CrossRef]

20. Nandi, S.K.; Rakete, S.; Nahomi, R.B.; Michel, C.; Dunbar, A.; Fritz, K.S.; Nagaraj, R.H. Succinylation is a gain-of-function modification in human lens $\alpha$ b-crystallin. Biochemistry 2019, 58, 1260-1274. [CrossRef]

21. Wladyka, B.; Piejko, M.; Bzowska, M.; Pieta, P.; Krzysik, M.; Mazurek, Ł.; Guevara-Lora, I.; Bukowski, M.; Sabat, A.J.; Friedrich, A.W.; et al. A peptide factor secreted by Staphylococcus pseudintermedius exhibits properties of both bacteriocins and virulence factors. Sci. Rep. 2015, 5, 14569. [CrossRef]

22. Nowakowski, M.; Jaremko, Ł.; Wladyka, B.; Dubin, G.; Ejchart, A.; Mak, P. Spatial attributes of the four-helix bundle group of bacteriocins-The high-resolution structure of BacSp222 in solution. Int. J. Biol. Macromol. 2018, 107, 2715-2724. [CrossRef]

23. Mak, P. Staphylococcal bacteriocins. In Pet-to-Man Traveling Staphylococci: A World in Progress; Savini, V., Ed.; Elsevier: Amsterdam, The Netherlands, 2018.

24. Pieta, P.; Majewska, M.; Su, Z.; Grossutti, M.; Wladyka, B.; Piejko, M.; Lipkowski, J.; Mak, P. Physicochemical studies on orientation and conformation of a new bacteriocin BacSp222 in a planar phospholipid bilayer. Langmuir 2016, 32, 5653-5662. [CrossRef]

25. Cross, A.S. What is a virulence factor? Crit. Care 2008, 12. [CrossRef]

26. Briaud, P.; Carroll, R.K. Extracellular vesicle biogenesis and functions in gram-positive bacteria. Infect. Immun. 2020, 88. [CrossRef]

27. Perez, R.H.; Zendo, T.; Sonomoto, K. Circular and leaderless bacteriocins: Biosynthesis, mode of action, applications, and prospects. Front. Microbiol. 2018, 9, 2085. [CrossRef]

28. Xu, H.D.; Shi, S.P.; Wen, P.P.; Qiu, J.D. SuccFind: A novel succinylation sites online prediction tool via enhanced characteristic strategy. Bioinformatics 2015, 31, 3748-3750. [CrossRef] 
29. Jia, J.; Liu, Z.; Xiao, X.; Liu, B.; Chou, K.C. PSuc-Lys: Predict lysine succinylation sites in proteins with PseAAC and ensemble random forest approach. J. Theor. Biol. 2016, 394, 223-230. [CrossRef]

30. Jia, J.; Liu, Z.; Xiao, X.; Liu, B.; Chou, K.C. ISuc-PseOpt: Identifying lysine succinylation sites in proteins by incorporating sequence-coupling effects into pseudo components and optimizing imbalanced training dataset. Anal. Biochem. 2016, 497, 48-56. [CrossRef]

31. Hasan, M.; Kurata, H. GPSuc: Global prediction of generic and species-specific succinylation sites by aggregating multiple sequence features. PLOS ONE 2018, 13, e0200283. [CrossRef]

32. Hasan, M.M.; Yang, S.; Zhou, Y.; Mollah, M.N.H. SuccinSite: A computational tool for the prediction of protein succinylation sites by exploiting the amino acid patterns and properties. Mol. BioSyst. 2016, 12, 786-795. [CrossRef] [PubMed]

33. Ning, Q.; Zhao, X.; Bao, L.; Ma, Z.; Zhao, X. Detecting succinylation sites from protein sequences using ensemble support vector machine. BMC Bioinform. 2018, 19, 237. [CrossRef]

34. Xu, Y.; Ding, Y.X.; Ding, J.; Lei, Y.H.; Wu, L.Y.; Deng, N.Y. ISuc-PseAAC: Predicting lysine succinylation in proteins by incorporating peptide position-specific propensity. Sci. Rep. 2015, 5, 10184. [CrossRef]

35. Zeng, F.; Pang, H.; Chen, Y.; Zheng, H.; Li, W.; Ramanathan, S.; Hoare, R.; Monaghan, S.J.; Lin, X.; Jian, J. First succinylome profiling of Vibrio alginolyticus reveals key role of lysine succinylation in cellular metabolism and virulence. Front. Cell. Infect. Microbiol. 2021, 10, 626574. [CrossRef] [PubMed]

36. Cochrane, S.A.; Surgenor, R.R.; Khey, K.M.W.; Vederas, J.C. Total synthesis and stereochemical assignment of the antimicrobial lipopeptide cerexin A1. Org. Lett. 2015, 17, 5428-5431. [CrossRef] [PubMed]

37. Fuchs, S.W.; Jaskolla, T.W.; Bochmann, S.; Kötter, P.; Wichelhaus, T.; Karas, M.; Stein, T.; Entian, K.D. Entianin, a novel subtilin-like lantibiotic from Bacillus subtilis subsp. spizizenii DSM 15029T with high antimicrobial activity. Appl. Environ. Microbiol. 2011, 77, 1698-1707. [CrossRef] [PubMed]

38. Bowers, A.A.; Walsh, C.T.; Acker, M.G. Genetic Interception and structural characterization of thiopeptide cyclization precursors from Bacillus cereus. J. Am. Chem. Soc. 2010, 132, 12182-12184. [CrossRef] [PubMed]

39. Roberts, R.E.; Hallett, M.B. Neutrophil cell shape change: Mechanism and signalling during cell spreading and phagocytosis. Int. J. Mol. Sci. 2019, 20, 1383. [CrossRef]

40. Ohba, H.; Nishikawa, M.; Kimura, M.; Yamasaki, N.; Moriwaki, S.; Itoh, K. Cytotoxicity Induced by Erythrina variegata serine proteinase inhibitors in tumor hematopoietic stem cell lines. Biosci. Biotechnol. Biochem. 1998, 62, 1166-1170. [CrossRef]

41. Simon, E.J.; Shemin, D. The preparation of s-succinyl coenzyme A. J. Am. Chem. Soc. 1953, 75. [CrossRef]

42. Sadhukhan, S.; Liu, X.; Ryu, D.; Nelson, O.D.; Stupinski, J.A.; Li, Z.; Chen, W.; Zhang, S.; Weiss, R.S.; Locasale, J.W.; et al. Metabolomics-assisted proteomics identifies succinylation and SIRT5 as important regulators of cardiac function. Proc. Natl. Acad. Sci. USA 2016, 113, 4320-4325. [CrossRef]

43. Wagner, G.R.; Payne, R.M. Widespread and enzyme-independent $\mathrm{N} \varepsilon$-acetylation and $\mathrm{N} \varepsilon$-succinylation of proteins in the chemical conditions of the mitochondrial matrix. J. Biol. Chem. 2013, 288, 29036-29045. [CrossRef]

44. O'Neil, M.J. The Merck Index: An Encyclopedia of Chemicals, Drugs, and Biologicals, 15th ed.; Royal Society of Chemistry: Cambridge, UK, 2013.

45. Bonar, E.; Bukowski, M.; Chlebicka, K.; Madry, A.; Bereznicka, A.; Kosecka-Strojek, M.; Dubin, G.; Miedzobrodzki, J.; Mak, P.; Wladyka, B. Human skin microbiota-friendly lysostaphin. Int. J. Biol. Macromol. 2021, 183, 852-860. [CrossRef]

46. Aasen, I.M.; Møretrø, T.; Katla, T.; Axelsson, L.; Storrø, I. Influence of complex nutrients, temperature and ph on bacteriocin production by Lactobacillus sakei CCUG 42687. Appl. Microbiol. Biotechnol. 2000, 53, 159-166. [CrossRef]

47. Padan, E.; Bibi, E.; Ito, M.; Krulwich, T.A. Alkaline PH homeostasis in bacteria: New insights. Biochim. Biophys. Acta Biomembr. 2005, 1717, 67-88. [CrossRef]

48. Krulwich, T.A.; Sachs, G.; Padan, E. Molecular aspects of bacterial PH sensing and homeostasis. Nat. Rev. Microbiol. 2011, 9, 330-343. [CrossRef]

49. Vallari, D.S.; Jackowski, S.; Rock, C.O. Regulation of pantothenate kinase by coenzyme A and its thioesters. J. Biol. Chem. 1987, 262, 2468-2471. [CrossRef]

50. Kosono, S.; Tamura, M.; Suzuki, S.; Kawamura, Y.; Yoshida, A.; Nishiyama, M.; Yoshida, M. Changes in the acetylome and succinylome of bacillus subtilis in response to carbon source. PLoS ONE 2015, 10, e0131169. [CrossRef]

51. Shibuta, S.; Mashimo, T.; Ohara, A.; Zhang, P.; Yoshiya, I. Intracerebroventricular administration of a nitric oxide-releasing compound, NOC-18, produces thermal hyperalgesia in rats. Neurosci. Lett. 1995, 187, 103-106. [CrossRef]

52. Grosser, M.R.; Paluscio, E.; Thurlow, L.R.; Dillon, M.M.; Cooper, V.S.; Kawula, T.H.; Richardson, A.R. Genetic requirements for Staphylococcus aureus nitric oxide resistance and virulence. PLoS Pathog. 2018, 14, e1006907. [CrossRef]

53. Wishart, D.S.; Bigam, C.G.; Yao, J.; Abildgaard, F.; Dyson, H.J.; Oldfield, E.; Markley, J.L.; Sykes, B.D. 1H, 13C and 15N chemical shift referencing in biomolecular NMR. J. Biomol. NMR 1995, 6, 135-140. [CrossRef] [PubMed]

54. Braunschweiler, L.; Ernst, R.R. Coherence transfer by isotropic mixing: Application to proton correlation spectroscopy. J. Magn. Reason. 1983, 53, 521-528. [CrossRef]

55. Jeener, J.; Meier, B.H.; Bachmann, P.; Ernst, R.R. Investigation of exchange processes by two-dimensional NMR spectroscopy. J. Chem. Phys. 1979, 71, 4546-4553. [CrossRef]

56. Bodenhausen, G.; Ruben, D.J. Natural abundance nitrogen-15 NMR by enhanced heteronuclear spectroscopy. Chem. Phys. Lett. 1980, 69, 185-189. [CrossRef] 
57. Delaglio, F.; Grzesiek, S.; Vuister, G.W.; Zhu, G.; Pfeifer, J.; Bax, A. NMRPipe: A multidimensional spectral processing system based on UNIX pipes. J. Biomol. NMR 1995, 6, 277-293. [CrossRef]

58. Goddard, T.D.; Kneller, D.G. Sparky—NMR Assignment and Integration Software. Available online: https://www.cgl.ucsf.edu/ home/sparky/ (accessed on 9 June 2021).

59. Güntert, P.; Mumenthaler, C.; Wüthrich, K. Torsion angle dynamics for NMR structure calculation with the new program dyana J. Mol. Biol. 1997, 273, 283-298. [CrossRef]

60. Shen, Y.; Bax, A. Protein backbone and sidechain torsion angles predicted from NMR chemical shifts using artificial neural networks. J. Biomol. NMR 2013, 56, 227-241. [CrossRef]

61. Schwieters, C.D.; Kuszewski, J.J.; Tjandra, N.; Marius Clore, G. The Xplor-NIH NMR molecular structure determination package J. Magn. Reason. 2003, 160, 65-73. [CrossRef]

62. Rosato, A.; Tejero, R.; Montelione, G.T. Quality assessment of protein NMR structures. Curr. Opin. Struct. Biol. 2013, 23, 715-724. [CrossRef]

63. Micsonai, A.; Wien, F.; Kernya, L.; Lee, Y.H.; Goto, Y.; Réfrégiers, M.; Kardos, J. Accurate secondary structure prediction and fold recognition for circular dichroism spectroscopy. Proc. Natl. Acad. Sci. USA 2015, 112, E3095-E3103. [CrossRef]

64. Micsonai, A.; Wien, F.; Bulyáki, É.; Kun, J.; Moussong, É.; Lee, Y.H.; Goto, Y.; Réfrégiers, M.; Kardos, J. BeStSel: A web server for accurate protein secondary structure prediction and fold recognition from the circular dichroism spectra. Nucleic Acids Res. 2018, 46, W315-W322. [CrossRef]

65. Wladyka, B.; Wielebska, K.; Wloka, M.; Bochenska, O.; Dubin, G.; Dubin, A.; Mak, P. Isolation, biochemical characterization, and cloning of a bacteriocin from the poultry-associated Staphylococcus aureus strain CH-91. Appl. Microbiol. Biotechnol. 2013, 97, 7229-7239. [CrossRef]

66. Diribe, O.; Thomas, S.; AbuOun, M.; Fitzpatrick, N.; la Ragione, R. Genotypic relatedness and characterization of Staphylococcus pseudintermedius associated with post-operative surgical infections in dogs. J. Med. Microbiol. 2015, 64, 1074-1081. [CrossRef]

67. AAT Bioquest. Four Parameter Logistic (4PL) Curve Calculator. Available online: https://www.aatbio.com/tools/fourparameter-logistic-4pl-curve-regression-online-calculator (accessed on 20 April 2021). 